\title{
ER-associated degradation is required for vasopressin prohormone processing and systemic water homeostasis
}

\author{
Guojun Shi, ${ }^{1}$ Diane R.M. Somlo, ${ }^{2}$ Geun Hyang Kim, ${ }^{1}$ Cristina Prescianotto-Baschong, ${ }^{3}$ Shengyi Sun, ${ }^{2}$ Nicole Beuret, ${ }^{3}$ \\ Qiaoming Long, ${ }^{4}$ Jonas Rutishauser, ${ }^{3}$ Peter Arvan, ${ }^{1,5}$ Martin Spiess, ${ }^{3}$ and Ling $Q \mathbf{i}^{1,5}$ \\ 'Department of Molecular and Integrative Physiology, University of Michigan Medical School, Ann Arbor, Michigan, USA. Division of Nutritional Sciences, Cornell University, Ithaca, New York, USA. \\ ${ }^{3}$ Biozentrum, University of Basel, Basel, Switzerland. . ${ }^{4}$ Cam-Su Mouse Genomic Resources Center, Suzhou University, Suzhou, Jiangsu, China. ${ }^{5}$ Division of Metabolism, Endocrinology and Diabetes, \\ Department of Internal Medicine, University of Michigan Medical School, Ann Arbor, Michigan, USA.
}

\begin{abstract}
Peptide hormones are crucial regulators of many aspects of human physiology. Mutations that alter these signaling peptides are associated with physiological imbalances that underlie diseases. However, the conformational maturation of peptide hormone precursors (prohormones) in the ER remains largely unexplored. Here, we report that conformational maturation of proAVP, the precursor for the antidiuretic hormone arginine-vasopressin, within the ER requires the ER-associated degradation (ERAD) activity of the Sel1L-Hrd1 protein complex. Serum hyperosmolality induces expression of both ERAD components and proAVP in AVP-producing neurons. Mice with global or AVP neuron-specific ablation of Se1L-Hrd1 ERAD progressively developed polyuria and polydipsia, characteristics of diabetes insipidus. Mechanistically, we found that ERAD deficiency causes marked ER retention and aggregation of a large proportion of all proAVP protein. Further, we show that proAVP is an endogenous substrate of Sel1L-Hrd1 ERAD. The inability to clear misfolded proAVP with highly reactive cysteine thiols in the absence of Sel1L-Hrd1 ERAD causes proAVP to accumulate and participate in inappropriate intermolecular disulfide-bonded aggregates, promoted by the enzymatic activity of protein disulfide isomerase (PDI). This study highlights a pathway linking ERAD to prohormone conformational maturation in neuroendocrine cells, expanding the role of ERAD in providing a conducive ER environment for nascent proteins to reach proper conformation.
\end{abstract}

\section{Introduction}

A wide variety of biologically active peptide hormones and neuropeptides regulate vital aspects of physiology, including food intake, body temperature, physical activity, and water balance. These signaling peptides are produced and activated through a unique intracellular process, whereby they are synthesized in the ER as prohormones (inactive or less active precursors) and are later activated through well-defined proteolytic cleavage(s) for storage in secretory granules (1). Pointing to the significance of ER-localized protein folding in this process, mutations that cause prohormone misfolding and ER retention have been linked to the pathogenesis of human diseases. For instance, mutations in pro-argininevasopressin (proAVP) cause diabetes insipidus (2), and mutations in proinsulin cause mutant INS gene-induced diabetes of youth (3). However, for the majority of prohormones, the mechanisms influencing prohormone folding in the ER remain largely undefined.

Diabetes insipidus is a condition in which the kidneys excrete an abnormally large volume of dilute urine, resulting in excessive urination (polyuria) and thirst (polydipsia) (4). Diabetes insipi-

\section{Related Commentary: p. 3591}

Authorship note: G. Shi, D.R.M. Somlo, and G.H. Kim contributed equally to this work Conflict of interest: The authors have declared that no conflict of interest exists. Submitted: April 27, 2017; Accepted: July 26, 2017.

Reference information: J Clin Invest. 2017;127(10):3897-3912.

https://doi.org/10.1172/JCI94771. dus is caused by either deficiency of the antidiuretic nonapeptide hormone AVP in the blood (i.e., central or neurogenic diabetes insipidus), or by a defective renal response to this hormone (i.e., nephrogenic diabetes insipidus) (4). AVP is synthesized in the ER as the 145-amino acid prohormone proAVP and then is cleaved to the final AVP nonapeptide in post-Golgi compartments for release into the circulation via the posterior pituitary gland. The majority of congenital neurogenic (central) diabetes insipidus cases occur as an autosomal-dominant disease, whereby any one of dozens of mutations in the AVP gene (e.g., G57S or $\triangle \mathrm{E} 47$ ) cause ER retention of proAVP and the formation of fibrillar aggregates $(2,5-8)$. Recent studies have shown that activation of autophagy and autophagy-associated neuronal death occur in response to proAVP aggregation at later stages of diabetes insipidus in mouse models subjected to intermittent water deprivation (9); however, the molecular mechanisms underlying physiological and pathophysiological proAVP folding and degradation remain to be explored. While both WT and mutant proAVP are subjected to proteasomal degradation (10), the nature and mechanisms of the degradative machinery (and, more importantly, the significance of this process in normal physiology and disease initiation) are totally unknown.

Misfolded proteins in the ER are targeted for proteasomal degradation by a highly conserved quality-control mechanism known as ER-associated degradation (ERAD) $(11,12)$. Among over a dozen E3 ligases in mammalian ERAD, the hydroxymethylglutaryl-CoA reductase degradation protein 1 (Hrd1) is a principal ER-resident E3 ligase that forms a complex with the ER-resident 
suppressor-enhancer of lin-12-like (Sel1L, also known as mammalian Hrd3) (13-19). Together, this complex is responsible for the degradation of a subset of misfolded proteins in the ER (11, 12). Like its yeast counterpart Hrd3p (14), mammalian Sel1L is required for the stability of $\mathrm{Hrd} 1$ (20) and may both directly recruit substrates to Hrd1 (21) and regulate Hrd1 activity (22). Germline Sel1L or Hrd1 deficiency is embryonically lethal in mice $(23,24)$, and acute global loss of Sel1L or Hrd1 in adult mice leads to premature death within 2 to 3 weeks $(20,23)$, suggesting that Sel1L and Hrd1 are indispensable for both development and postnatal survival (11). Recent studies of cell type-specific Sel1L-Hrd1 deficiency in adipocytes $(25,26)$, B cells $(27)$, and colonic epithelium (21) have delineated the physiological importance of ERAD in these various tissues and identified several endogenous substrates (11), including IRE1 $\alpha$ of the unfolded protein response (UPR) in many cell types (20); lipoprotein lipase and PGC1 $\beta$ in adipocytes (25, 26); and pre-B cell receptor in developing B cells (27). However, the role of ERAD in neuroendocrine cells has not been explored.

A major posttranslational modification of secretory proteins in the ER is disulfide bond formation involving oxidation of a pair of cysteine residues (28), which is catalyzed by enzymes of the protein disulfide isomerase (PDI) family. PDI, the founding member of this family and a major oxidoreductase in the ER lumen, has a broad substrate range (29) and catalyzes oxidative folding and isomerization of many substrates (30). In its catalytic cycle involving sequential oxidation and reduction reactions, PDI forms transient mixed disulfide bonds with substrates through its active Cys-X-X-Cys thioredoxin motif (Cys, cysteine; $\mathrm{X}$, any amino acids) (31). PDI can act as a protein chaperone to help protein folding and stability $(30,32)$ by interacting with substrates at different stages of the folding process, i.e., unfolded, partially folded, or native state (32), and also has a role in ERAD. Recent studies have suggested that PDI in the ER is able to reduce proinsulin disulfide bonds (33) and in this way may prime proinsulin Akita-mutant protein to unfold for Sel1L-Hrd1 ERAD (3). Adding further complexity to the function of PDI, another study suggested that the role of PDI in ERAD may be substrate dependent (34).

In the characterization of inducible Sel1L-KO mice (20), we made a serendipitous discovery that these animals develop central, not nephrogenic, diabetes insipidus. Strikingly, AVP neuron-specific Sel1L-deficient mice phenocopy inducible Sel1L-KO mice in terms of diabetes insipidus. We found that mammalian Sel1L-Hrd1 ERAD degrades misfolded WT proAVP in AVP neurons under physiological conditions and thus plays a vital role in systemic water balance. If not degraded effectively, misfolded proAVP molecules accumulate and participate in inappropriate intermolecular disulfide-bonded fibrillar aggregates, a process promoted by the redox activity of PDI.

\section{Results}

Mice with acutely induced global Sel1L deletion develop central diabetes insipidus. We previously generated a mouse model of inducible global Sel1L KO $\left(S e l 1 L^{f / f} E R C r e^{+}\right.$, herein designated Sel1L $\left.L^{E R C r e}\right)$, in which the Sel1L exon 6 flanked by loxP sites was excised by ER-Cre, a tamoxifen-activated, estrogen receptor-Cre (ERCre) fusion protein expressed globally under the control of the actin promoter (20). In the characterization of this mouse model, we made a ser- endipitous observation that the bedding in Sel1 $L^{E R C r e}$ cages became conspicuously wet within days (red circle and arrows, Figure 1A). Indeed, further analysis of Sel1L $L^{E R C r e}$ mice confirmed that they developed increased water intake (i.e., polydipsia) (Figure 1, B and C), elevated urine output (i.e., polyuria) (Figure 1D), and decreased urine osmolality (Figure 1E) compared with WT (Sel1L $\left.L^{f / f l}\right)$ littermates. This phenotype was consistent with diabetes insipidus (2) and, strikingly, manifested within 3 days of the first tamoxifen injection and deteriorated thereafter (Figure 1, C and D).

To determine whether Sel1L ${ }^{\text {ERCre }}$ mice developed central or nephrogenic diabetes insipidus, we treated Sel1L ${ }^{E R C r e}$ mice with 1-deamino-8-arginine vasopressin (dDAVP), a synthetic agonist of renal vasopressin receptors (35). dDAVP injection triggered the translocation of Aquaporin 2 water channels to the apical membrane of kidney collecting duct cells (Supplemental Figure 1A; supplemental material available online with this article; https://doi. org/10.1172/JCI94771DS1) in both Sel1L ${ }^{f l / f l}$ and Sel1L ${ }^{\text {ERCre }}$ mice (Figure 1F). Further, dDAVP injection significantly increased the urine osmolality of both Sel1L $L^{f l f l}$ and Sel1 $L^{E R C r e}$ mice but had a significantly greater effect on Sel1L ${ }^{E R C r e}$ mice, increasing urine osmolality to a level comparable to that of WT mice (Figure 1G). This robust renal response to dDAVP stimulation in Sel1 $L^{E R C r e}$ mice suggested that their renal urine-concentrating ability was intact. Providing further support for this idea, urine protein, glucose, ketone, bilirubin, and $\mathrm{pH}$ were comparable between $S e l 1 L^{E R C r e}$ and $S e l 1 L^{f l / f l}$ mice (Supplemental Table 1). Kidney weights (not shown) and gross structure of kidneys (Supplemental Figure 1B) were comparable as well. These data point to a central defect as the cause of diabetes insipidus in Sel1L ${ }^{E R C r e}$ mice. Indeed, the level of circulating AVP the antidiuretic hormone - was significantly decreased in Sel1L $L^{\text {ERCre }}$ mice (Figure 1H). Hence, we conclude that acute loss of Sel1L in adult mice leads to the development of central diabetes insipidus.

Sel1L-Hrd1 ERAD expression is enriched in AVP neurons and responsive to hyperosmolality. Both Sel1L and Hrd1 transcripts are ubiquitously expressed throughout the body (20) (Supplemental Figure 2A). Immunofluorescence staining of brain sections revealed that Hrd1 is expressed in many regions of the brain (Figure 2A and Supplemental Figure 2B) and highly enriched in the hypothalamic supraoptic nucleus (SON) and paraventricular nucleus (PVN) regions (Figure 2, B and C) -2 regions that contain magnocellular, AVP-producing neurons. Costaining with Hrd1 and AVP revealed that, indeed, Hrd1 is enriched in AVP neurons (Figure 2, D and E). Quantitative analysis of immunofluorescence signal intensity in individual AVP neurons further demonstrated a positive correlation between protein levels of AVP and Hrd1 in AVP neurons (Figure 2, D and E). This observation led us to examine Hrd1 expression in AVP neurons under hyperosmolality conditions. Hyperosmolality caused by either water deprivation for 3 days or salt loading ( $2 \%$ sodium chloride drinking water) for 7 days not only induced AVP but also significantly elevated Hrd1 protein levels in the AVP neurons (Figure 2, E-G, and quantitated in Figure $2 \mathrm{H}$ ). Hence, Sel1L-Hrd1 ERAD expression in AVP neurons is responsive to serum hyperosmolality.

Mice with AVP neuron-specific Sel1L deficiency develop central diabetes insipidus. To investigate the role of Sel1L-Hrd1 ERAD in AVP neurons, we generated AVP neuron-specific Sel1L-KO $\left(\right.$ Sel1 $\left.{ }^{A V P}\right)$ mice by crossing Sel1L $L^{f l f l}$ mice with AVP neuron-specific 
A

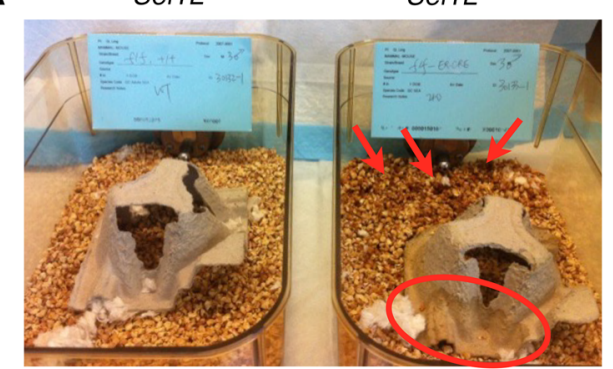

D
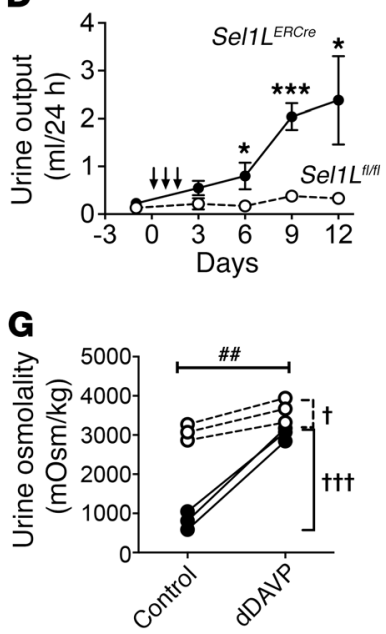

B

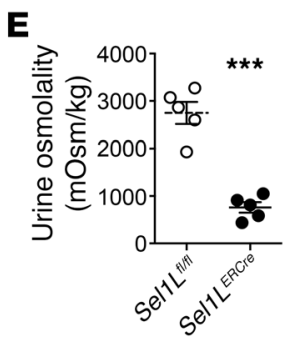

H

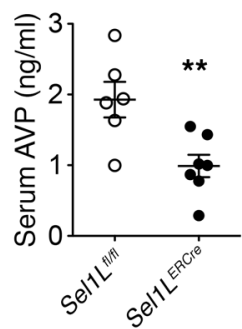

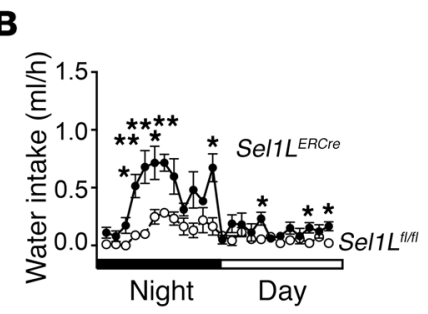

C

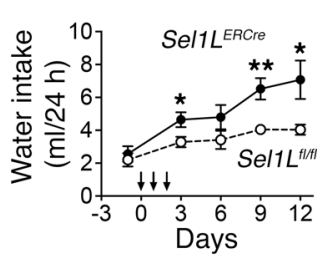

$\mathbf{F}$
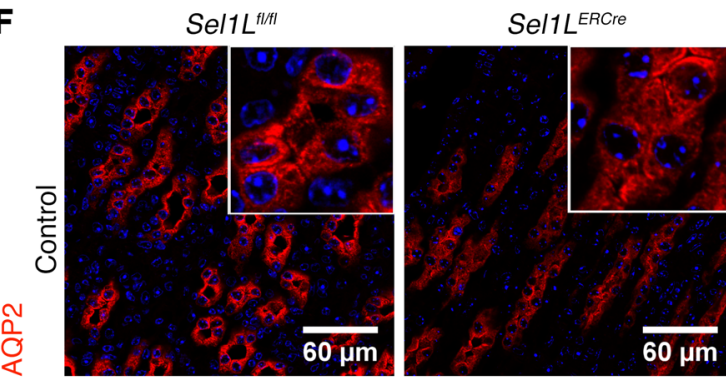

$\overline{\underline{\alpha}}$
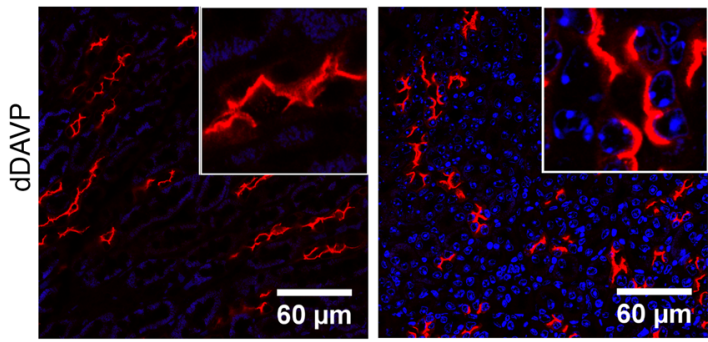

Figure 1. Mice with globally induced Sel1L deficiency develop central diabetes insipidus. (A) Representative images of cages housing WT (Sel1L/f) and inducible KO (Sel1L $\left.{ }^{E R C r e}\right)$ mice 3 days after changing to fresh bedding. Circle and arrows indicate the wet spot. Arrows indicate damp bedding. (B) Metabolic cage analysis of 24-hour water intake by Sel1 ${ }^{f l f l}$ and Sel1L ERCre mice on day 11 or 12 after tamoxifen administration ( $n=4$ mice/group). (C and $\left.\mathbf{D}\right)$ Progressive changes in water intake (C) and urine output (D) in Sel1 $L^{f l / f l}$ and Sel1L ${ }^{E R C r e}$ mice after tamoxifen treatment (indicated by 3 arrows). $n=6$ mice each group. (E) Urine osmolality 2 weeks after tamoxifen treatment. (F) Representative fluorescence images of Aquaporin 2 (AQP2, red) in the kidney collecting duct cells of Sel1 ${ }^{f l / f l}$ and Sel1L ${ }^{\text {ERCre }}$ mice 1 week after tamoxifen treatment, either before (control) or 30 minutes after dDAVP injections. (G) Response to the AVP receptor agonist dDAVP in Sel1 $L^{f l f l}$ and Sel1 ERCre mice 2 weeks after tamoxifen administration. Urine osmolality before and after administration is shown ( $n=3$ each group). ${ }^{\dagger} P<0.05$, and ${ }^{\dagger+\dagger} P<0.001$, by paired Student's $t$ test, showing that both Sel1L ${ }^{f l / f l}$ and Sel1L ${ }^{E R C r e}$ mice responded to dDAVP with significant increases in osmolality. ${ }^{\# \# P}<0.01$, by 2 -way ANOVA analysis, showing a differential response to dDAVP in Sel1L ${ }^{f l / f l}$ and Sel1L ${ }^{E R C r e}$ mice. (H) Serum AVP levels 2 weeks after tamoxifen treatment. Values represent the mean \pm SEM. ${ }^{*} P<0.05,{ }^{* *} P<0.01$, and ${ }^{* * *} P<0.001$, by Student's $t$ test $(\mathbf{B}-\mathbf{E}$ and $\mathbf{H})$. Data represent at least 2 independent experiments.

Cre-driver mice. Sel1 $L^{f / f l}$ littermates without Cre were included as WT controls. Sel1 $L^{A V P}$ mice appeared grossly normal in terms of morphology and growth compared with WT mice (Figure 3A). Sel1 ${ }^{A V P}$ mice recapitulated the diabetes insipidus phenotype seen in global Sel1 $L^{E R C r e}$ mice, with increased water intake, increased urination (Figure 3, B and C), and decreased urine osmolality (Figure 3, D and E). Circulating AVP levels in Sel1L $L^{A V P}$ mice were decreased to $30 \%$ of WT control values (Figure $3 \mathrm{~F}$ ). Indeed, decreased urine osmolality in Sel1 $L^{A V P}$ mice was observed under ad-libitum water conditions as early as 3 weeks of age, i.e., at the time of weaning, in both males and females (Figure 3, D and E). Taken together, these findings establish a vital role of Sel1L in AVP neurons in the regulation of systemic water balance.

Sel1L deficiency in AVP neurons does not lead to neuronal death or loss. We next explored how Sel1L deficiency in the ER of AVP neurons leads to systemic water imbalance. As in other cell types (20, 21, 27), Hrd1 protein levels were markedly reduced in Sel1L-deficient AVP neurons (Figure 4A and quantitated in Supplemental Fig- ure 3A). At 1 week of age, Sel1 $L^{A V P}$ mice had comparable numbers of AVP neurons and comparable levels of Avp mRNA relative to levels in control Sel1L $L^{f / f l}$ mice, as demonstrated by ISH of Avp mRNA (Figure 4B). Likewise, Sel1L $L^{\text {ERCre }}$ mice had comparable AVP neuron numbers and Avp mRNA levels compared with the control cohorts (Supplemental Figure 3, B and C). In addition, there was no detectable cell death in Sel1L-deficient AVP neurons from Sel1L ${ }^{A V P}$ mice (Supplemental Figure 3D). Furthermore, AVP neuronal activation as marked by nuclear c-Fos $(36,37)$ was intact in Sel1L $L^{\text {ERCre }}$ mice compared with that observed in Sel1 $L^{f l f l}$ mice (Figure 4, C and D). While low abundance of AVP neurons in the hypothalamus precluded efforts to directly quantitate ER stress in these animal models using standard biochemical methods (Western blotting and quantitative PCR [qPCR]) (38), these data excluded the possibility of neuronal cell death or diminished responsiveness as major causes of disease initiation in Sel1L-deficient mice.

Sel1L deficiency causes intracellular ER retention of proAVP. As circulating AVP levels were decreased in the bloodstream of 

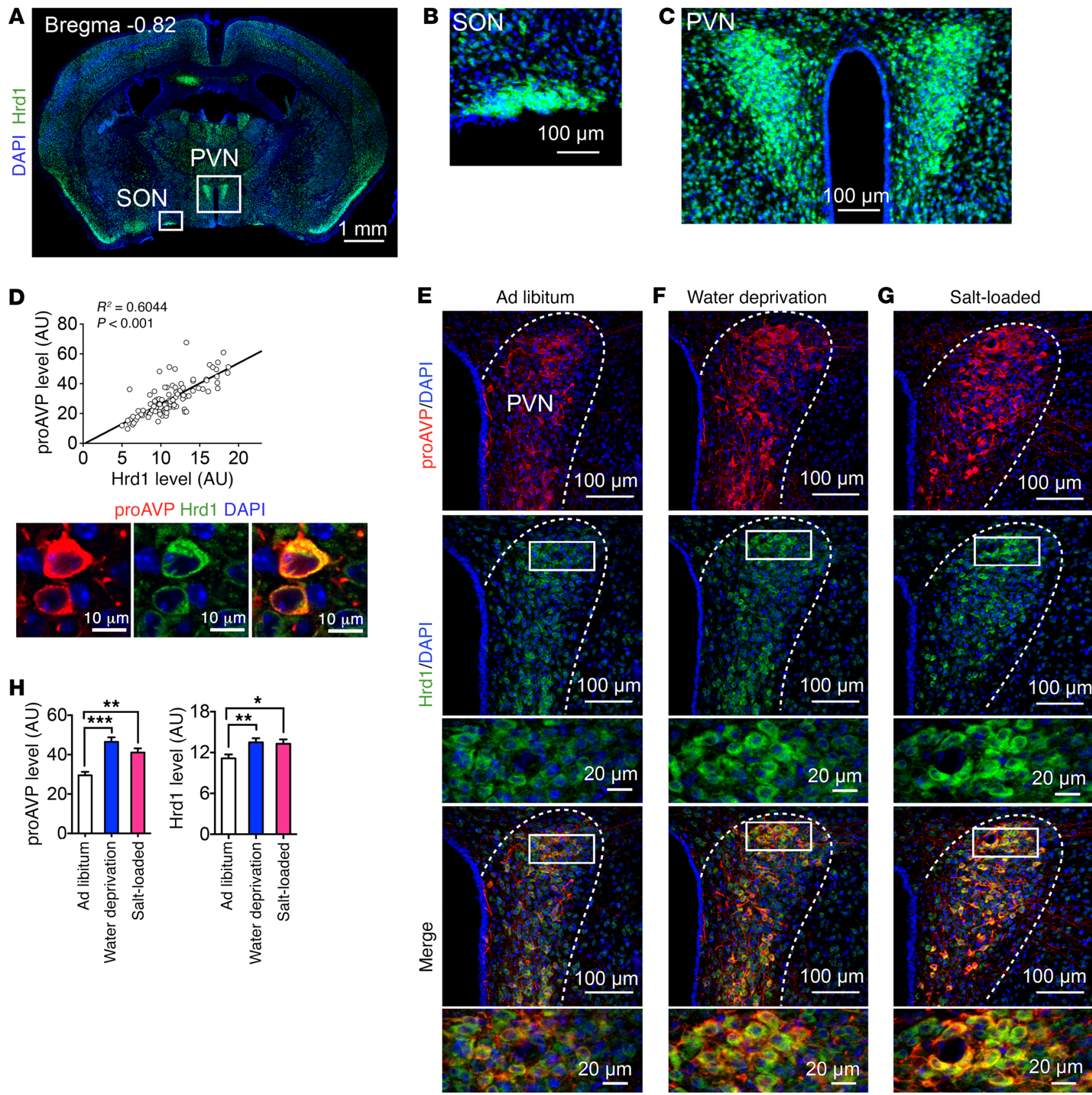

Figure 2. HRD1 is highly enriched in AVP neurons and upregulated during water deprivation. (A-C) Representative fluorescence images of Hrd1 expression in brain sections from WT mice with ad libitum access to water (A) and close-up view of the PVN (B) and SON (C). (D) Scatterplot demonstrating the relationship between Hrd1 and proAVP fluorescence mean intensity in single AVP neurons from WT mice with ad libitum access to water. (E-C) Representative fluorescence images of proAVP and Hrd1 in WT mice PVN under ad libitum access to water (E) or osmotic stress conditions. Mice were either water deprived for 72 hours (F) or given water containing $2 \%$ sodium chloride (salt-loaded) for 7 days (C). (H) Mean fluorescence intensity of proAVP and Hrd1 staining for images in E-G. (A-C) Representative images for 2 WT male mice; (D) 100 proAVP-positive neurons were quantitated. Scale bar: $10 \mu \mathrm{m}$. (E-G) $n=2$ mice/group; (H) $n=50$ AVP-positive cells from 2 mice per condition. Values represent the mean $\pm \mathrm{SEM} .{ }^{*} P<0.05,{ }^{*} P<0.01$, and ${ }^{* * *} P<0.001$, by Student's $t$ test. Data represent at least 2 independent experiments.

Sel1L $L^{A V P}$ mice, we next examined the level and distribution of proAVP protein in AVP neurons. As the disease manifested very early in life (Figure 3, D and E), we performed subsequent analysis using 1- to 3-week-old Sel1L ${ }^{A V P}$ mice. proAVP consists of the AVP nonapeptide, a carrier protein called Neurophysin II (NPII), and a glycopeptide (GP) (Figure 4E). proAVP is folded in the ER and is cleaved to final products in secretory granules as they travel down the axons toward synaptic terminals. We costained brain tissue sections with 2 different proAVP antibodies specifically recognizing AVP and NPII domains (Figure 4E) $(39,40)$ and found that at 1 week of age, there was already a notable reduction of AVP signal in the axons of Sel1L $L^{A V P}$ neurons (Figure $4 \mathrm{~F}$ and Supplemental Figure 4). Confirming these findings, we stained for proAVP in 3-week-old WT mice and found that AVP processing intermediates were evi- 
A
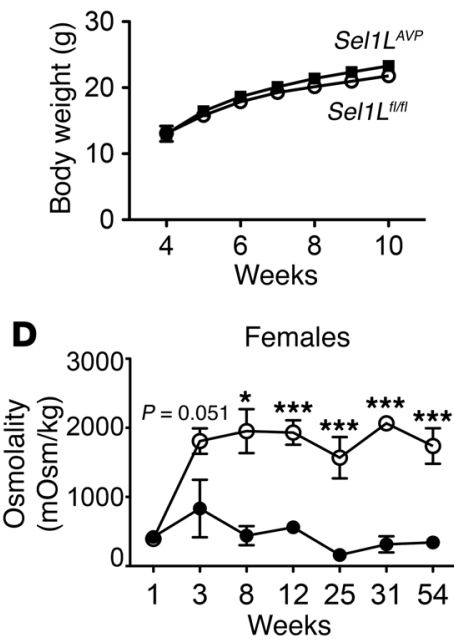
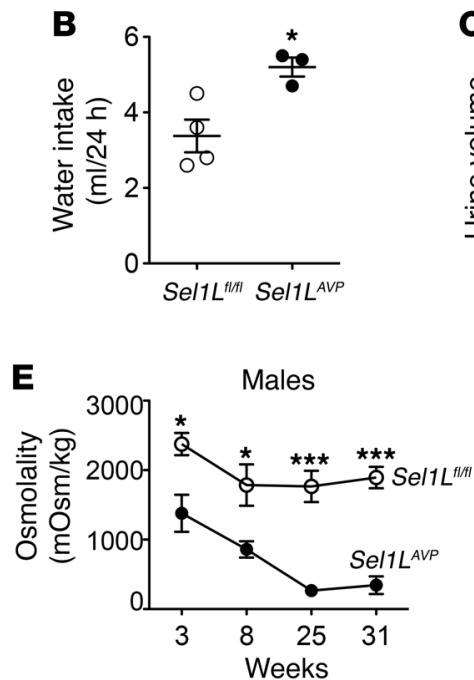
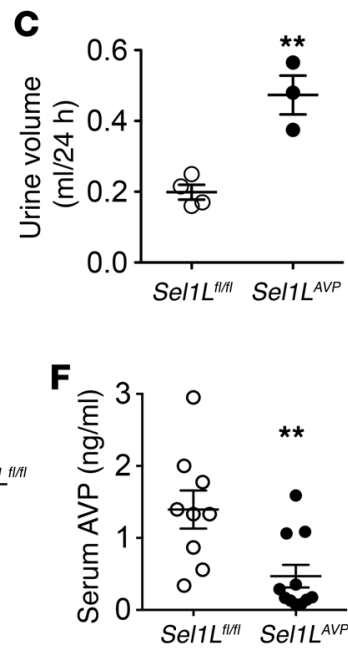

Figure 3. Mice with AVP neuron-specific Sel1L deficiency develop central diabetes insipidus. (A-E) Growth curve (A), water intake (B), urine output (C), and urine osmolality in female (D) and male (E) mice, and serum AVP levels (F) for WT (Sel1 $L^{f l / f l}$ ) and AVP neuron-specific Sel1L-KO (Sel1L ${ }^{\text {AVP }}$ ) mice. (A) $n=5$ male mice each; $(\mathbf{B}$ and $\mathbf{C}) n=3-4$ male mice each, aged 6-8 weeks; ( $\mathbf{D}$ and $\mathbf{E}) n=2-8$ mice for each time point; (F) $n=9-11$ mice each, aged 6-10 weeks. $P=0.051$, by Student's $t$ test. Both male and female mice were used. Values represent the mean \pm SEM. ${ }^{*} P<0.05$, ${ }^{* *} P<0.01$, and ${ }^{* * *} P<0.001$, by Student's $t$ test. Data represent at least 2 independent experiments.

of SEL1L destabilized HRD1 and led to HRD1 deficiency as shown previously (20), while HRD1 deletion stabilized SEL1L and led to SEL1L accumulation in both cell lines (Figure 5C). Loss of HRD1 decreased proAVP polyubiquitination (lane 3 vs. lane 2, Figure 5D) and increased its stability (Figure 5E), suggesting that SEL1L-HRD1 ERAD is responsible for the protein turnover of proAVP. Moreover, to determine the changes in detergent solubility of proAVP, we fractionated cell lysates into NP-40-soluble and -insoluble fractions. In line with proAVP being an endogenous substrate, loss of Sel1L or Hrd1 dramatically increased intracellular proAVP protein levels by over 4-fold in detergent-soluble fractions (Figure 5F). In addition,

dent in a "beads-on-a-string" axonal pattern, whereas this signal was markedly diminished in Sel1 $L^{A V P}$ axons (arrows, Figure $4 G$ ).

In the soma region of Sel1L-deficient neurons, proAVP protein exhibited a qualitatively altered localization with a more circular and perinuclear distribution pattern (Figure $4 \mathrm{H}$ ). Quantification of the percentage of proAVP immunofluorescence that colocalized with BiP immunofluorescence in individual cells was significantly increased (Figure 4I), suggesting a significant increase in the fraction of proAVP in the ER. Correlation of fluorophore intensity is considered another way to examine the colocalization of immunostained proteins (41); thus, we calculated the correlation between proAVP and BiP fluorescence intensity at each pixel as Pearson's coefficient ( $r$, ranges from -1 to +1 ), where +1 indicates a perfect positive correlation between the intensity of 2 fluorophores. Indeed, Pearson's coefficient was significantly increased in Sel1L ${ }^{A V P}$ neurons compared with that in WT control neurons, further supporting the idea of an altered distribution that features increased proAVP localization in the ER (Figure 4J). Of note, elevated BiP protein levels in the Sel1L ${ }^{A V P}$ neurons (Figure $4 \mathrm{H}$ ) was likely the result of an adaptive response to ERAD deficiency. Taken together, the data demonstrated that Sel1L deficiency causes ER retention of endogenous WT proAVP.

proAVP is an endogenous substrate of SEL1L-HRD1 ERAD. We next investigated mechanistically how Sel1L deficiency affects proAVP folding, degradation, and maturation in the ER. A previous study reported that both WT and mutant proAVP are degraded by proteasomes in the cytosol (10); however, the nature of the ERAD machinery responsible for proAVP protein turnover remains unknown. proAVP expressed in HEK293T cells in vitro coimmunoprecipitated with HRD1 (Figure 5A) and was ubiquitinated (Figure 5B). We then used the CRISPR/Cas9 system to generate SEL1L and HRD1-deficient human HEK293T cells and mouse neuroblastoma Neuro2A (N2a) cells (Figure 5C). Deletion
SEL1L-HRD1 ERAD also stimulated ubiquitination of the diseaseassociated mutant proAVP-G57S (lane 4 vs. lane 5, Figure 5D) and targeted both proAVP-G57S and $\triangle \mathrm{E} 47$ mutants for proteasomal degradation (Figure 5, G and $\mathrm{H}$ ). Hence, we conclude that both WT and some mutant proAVP are Sel1L-Hrd1 ERAD substrates.

SEL1L-HRD1 ERAD prevents the propagation of misfolded WT proAVP. Intriguingly, loss of Sel1L or Hrd1 also increased the abundance of NP-40-insoluble proAVP (Figure 5F), presumably in the form of protein aggregates. We sought to directly visualize intracellular retention and aggregation of proAVP. Confocal microscopy revealed that, unlike WT proAVP, proAVP-G57S was retained in the ER of WT N2a cells, as expected $(6,42)$ (Figure 6A and Supplemental Figure 5A). Similarly, in the absence of Sel1L, WT proAVP immunostained in a perinuclear distribution with a strong colocalization with BiP (Figure 6B and Supplemental Figure 5B).

We next performed Immunogold electron microscopic analysis of in vitro and in vivo samples. While WT proAVP was detected in the ER sheets of WT N2a cells (arrows, Figure 6C), WT proAVP in Sel1L-null N2a cells appeared to accumulate in amyloid-like fibrillar structures (Figure 6D). This pattern bore striking resemblance to that of mutant proAVP in WT and Sel1L-deficient N2a cells (Figure 6, C and D, $\triangle \mathrm{E} 47$ proAVP). Moreover, Immunogold coupled to a proAVP-specific antibody decorated large endogenous AVP aggregates in Sel1 $L^{A V P}$ neurons, unlike the small but dense AVP-containing vesicles in WT neurons (Figure 6, E and F). Thus, these data demonstrate that SEL1L-HRD1 ERAD helps to limit the ER retention and aggregation of WT proAVP.

To investigate the molecular basis for ERAD deficiency-triggered proAVP aggregation, we performed sucrose gradient fractionation of proAVP-containing protein complexes, followed by nonreducing SDS-PAGE and Western blot analyses. While WT proAVP formed predominantly monomers in WT cells, the proAVP-G57S mutant formed exclusively high-molecular-weight 


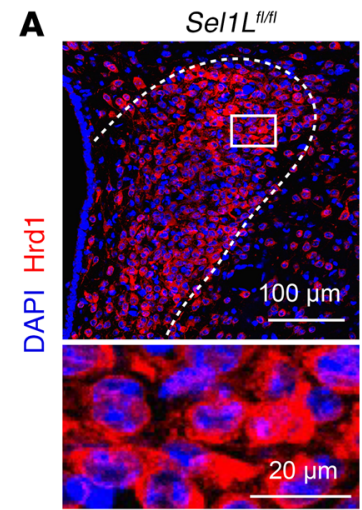

C

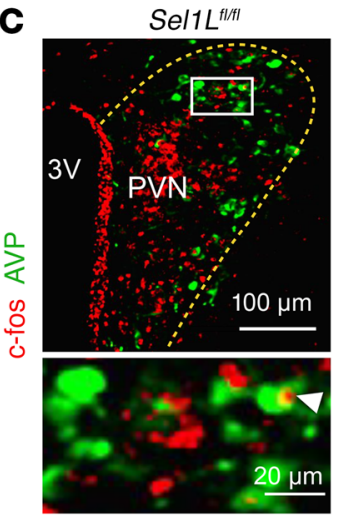

G

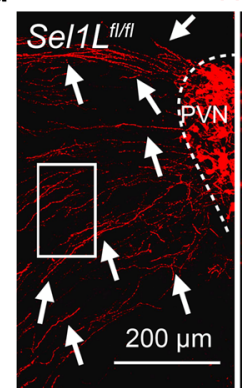

AVP
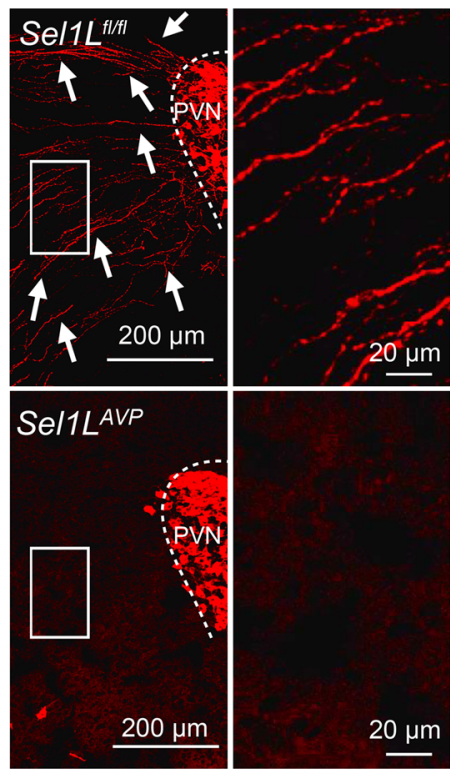

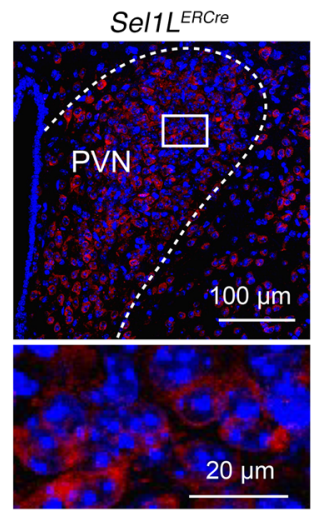

Sel1LERCre

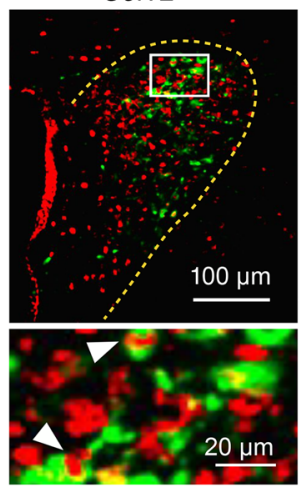

B

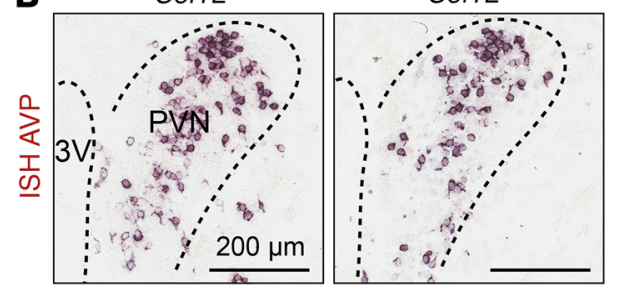

E
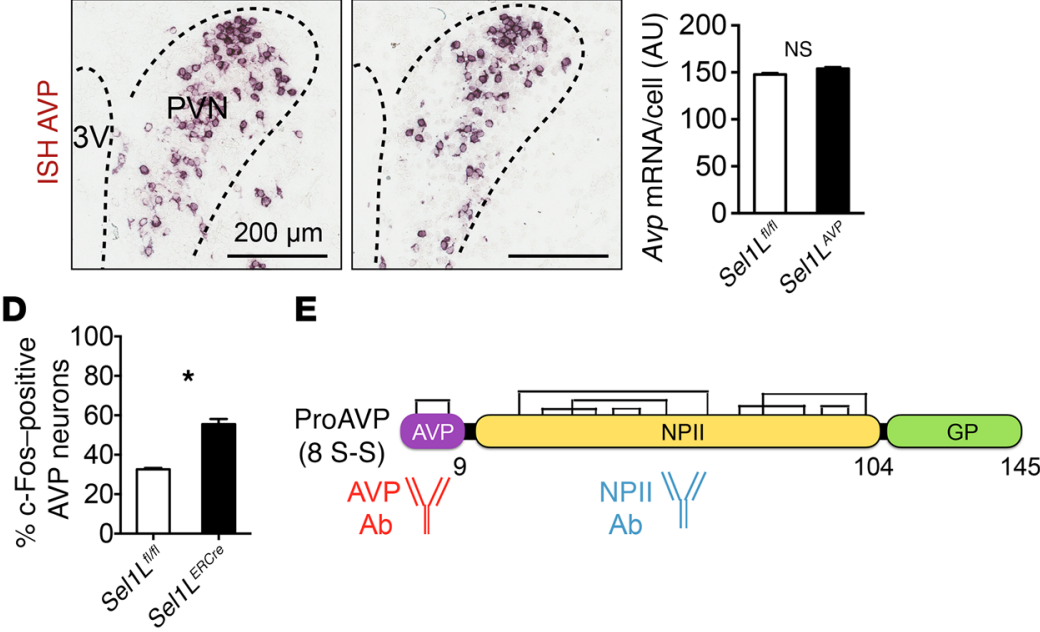

$\mathbf{F}$
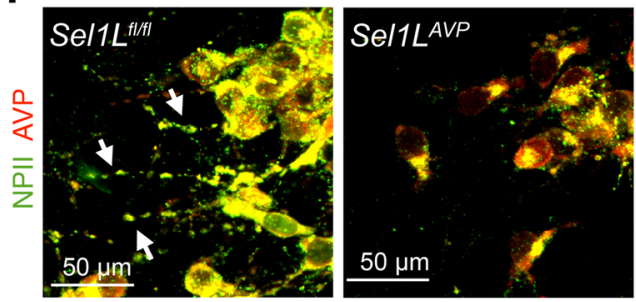

H

Sel1 $1 L^{f \prime \prime I}$

Sel1L AVP

I
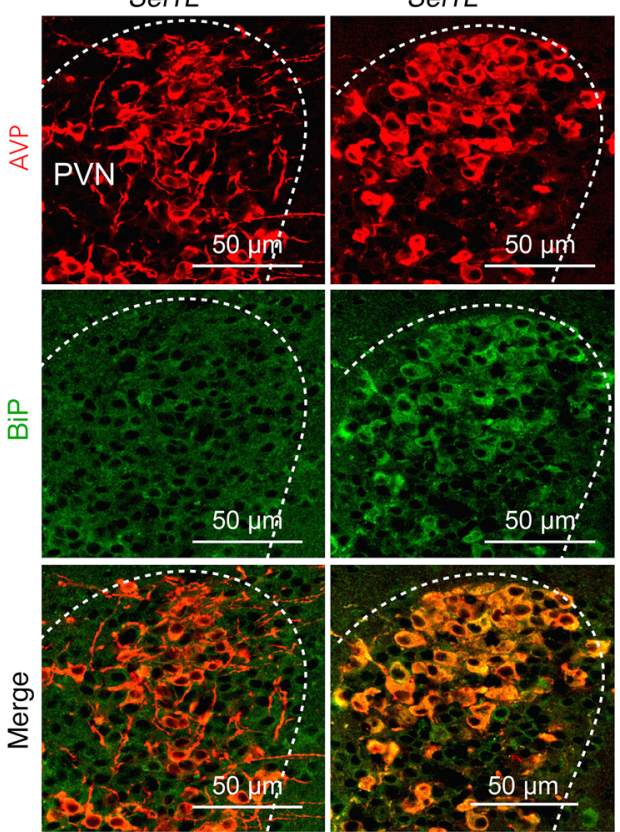

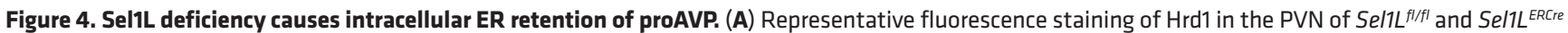
mice 6 days after tamoxifen injections. $n=3$ mice each genotype. Dashed line outlines the PVN. (B) ISH of Avp mRNA in the PVN region of Sel/L fl/fl and Sel/LAVP mice at 1 week, with quantification of probe hybridization intensity shown on the right. Dashed line traces the PVN. (C) Representative fluorescence images

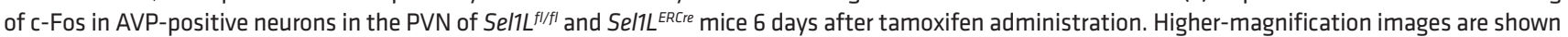
below. 3V, third ventricle. (D) Quantification of the percentage of c-Fos-positive AVP neurons. $n=2$ female mice/genotype. (E) Schematic structure of proAVP protein and its processed products (AVP, NPII, and GP). Eight disulfide bonds and areas recognized by the AVP- and NPII-specific antibodies are shown. Amino acid positions for each polypeptide are shown. (F) Representative fluorescence images of proAVP in the PVN recognized by 2 antibodies specific for different regions of proAVP (AVP and NPII) in 1-week-old Sel/1 L ${ }^{f / f l}$ and Sel/1 LVP mice, showing largely absent axonal distribution of AVP-containing transport vesicles (arrows) in Sel1L AVP mice. (C) Representative fluorescence images of proAVP staining showing the absence of AVP-positive transport vesicles (arrows) in the axons of 3-week-old Sell1 AVP mice, with zoomed-in images on the right. $n=3$ mice/group. (H-J) Representative fluorescence images of proAVP and BiP costaining in 3-week-old mice (H). Quantification of colocalization and Pearson's coefficient between AVP and BiP staining (I and $\mathbf{J}) . n=3$ female mice/group. Values represent the mean \pm SEM. NS, not significant. ${ }^{*} P<0.05$, by Student's $t$ test. Data represent at least 2 independent experiments. 
aggregates, as expected (Figure 6G). When expressed together, the presence of the proAVP-G57S mutant resulted in the recruitment of coexpressed WT proAVP into high-molecular-weight complexes. These data are consistent with an autosomal-dominant effect of the mutant, and they also served as a positive control for the assay. Strikingly, in the absence of SEL1L-HRD1 ERAD, WT proAVP formed predominantly ( 90\%) high-molecular-weight complexes (Figure $6 \mathrm{H}$ ) in a pattern similar to that of WT cells coexpressing WT and mutant proAVP. Since this inability to prevent proAVP aggregation is linked to diminished circulating AVP with diabetes insipidus, these data demonstrate the profoundly important function of SEL1L-HRD1 ERAD not only to clear misfolded proAVP, but also to prevent the majority of WT proAVP from being recruited into protein aggregates.

$E R A D$ deficiency triggers proAVP aggregation via intermolecular disulfide bonds. In the ER, proAVP forms 8 intramolecular disulfide bonds (Figure 4E) and forms a noncovalent homodimer prior to ER exit (43). Pulse-chase analysis revealed that nascent proAVP proteins readily formed detergent (NP-40) soluble high-molecularweight complexes in ERAD-deficient cells within 15 minutes of the pulse (lane 4 vs. lane 1, Figure 7A and lane 1 vs. lane 5, Figure 7B), which appeared quite similar to the complexes formed in WT cells expressing proAVP-G57S (lane 4 vs. lane 5, Figure 7B). In line with the notion that proAVP-G57S is also an ERAD substrate, SEL1LHRD1 deficiency further increased the aggregation of proAVPG57S (lane 8 vs. lane 4, Figure 7B). These high-molecular-weight complexes in the NP-40-soluble fraction were sensitive to the reducing agent DTT and heat (Figure $7 \mathrm{~B}$ ), pointing to the involvement of disulfide bonds in the formation of these complexes.

Strikingly, a large proportion of newly synthesized proAVP ( $43 \%$ ) following a 15 -minute pulse was detected in the NP-40insoluble fractions of ERAD-deficient cells versus only approximately $5 \%$ in WT cells (lanes 10 to 4 vs. lanes 7 to 1, Figure 7A). Moreover, some of these insoluble aggregates were resistant to both SDS and heat (lanes 10-12, Figure 7A). These data suggest that nascent proAVP is being actively recruited to the aggregates in the absence of SEL1L-HRD1 ERAD. Indeed, when all 16 cysteines of proAVP were mutated to serine residues (named the "abcd" mutant [ref. 5]), the formation of protein aggregation for nascent proAVP was nearly abolished, even in ERAD-deficient cells (lane 5 vs. lane 6, Figure 7B), further supporting the role of intermolecular disulfide bonds in the formation of proAVP aggregates.

To provide further evidence for the importance of intermolecular disulfide bonds in mediating proAVP aggregation in ERAD-deficient cells, we coexpressed HA-tagged WT or mutant proAVP with Flag-tagged WT or mutant proAVP in HRD1-deficient HEK293T cells, followed by Western blot analysis under nonreducing conditions (Figure 7C). When both HA- and FLAG-tagged constructs expressed WT proAVP, high-molecular-weight complexes formed in ERAD-deficient cells (lanes 1 and 7). When HA-tagged proAVPG57S was coexpressed with FLAG-tagged WT proAVP, even larger aggregates were formed (lane 4 vs. lane 1 and lane 10 vs. lane 7).

To further increase the abundance of proAVP in the ER, we added a KDEL sequence to the C-terminus of WT proAVP to retain nascent proAVP proteins, presumably at different folding stages (44). Indeed, WT proAVP-KDEL augmented protein aggregation when coexpressed with WT proAVP in HRD1-deficient cells (lane 2 vs. lane 1 and lane 8 vs. lane 7, Figure 7C), pointing to the importance of intra-ER abundance of proAVP in the formation of aggregates. By contrast, WT proAVP-KDEL had little effect on the aggregation of proAVP-G57S, consistent with the dominant-negative effect of the disease mutant (lane 5 vs. lane 4 and lane 11 vs. lane 10). Importantly, coexpression of cysteine-less proAVP (proAVP-abcd) lessened aggregation of WT proAVP (lane 3 vs. lanes 1 and 2; lane 9 vs. lanes 7 and 8) and attenuated aggregation of the proAVP-G57S mutant (lane 6 vs. lanes 4 and 5 and lane 12 vs. lanes 10 and 11), providing further support for the notion that proAVP aggregation in the ER is mediated through disulfide bonds. Thus, we conclude that in the absence of ERAD, misfolded proAVP molecules are not cleared and therefore accumulate and participate in erroneous intermolecular disulfide-bonded aggregates.

Enzymatic activity of PDI is required for proAVP degradation. Studies have shown that PDI, but not other members of the PDI family (ERp57 and ERdj5), is able to participate in the ERAD of misfolded Akita proinsulin by the Sel1L-Hrd1 complex (3). We next addressed the relationship between PDI and proAVP. PDI interacted with WT proAVP (Figure 8A) and formed heterodimeric intermediates with proAVP via disulfide bonds (Figure 8B) in WT HEK293T cells. To further demonstrate that the interaction between PDI and proAVP requires PDI redox enzymatic activity, we used the PDI-C56A "trap mutant," whereby the active CXXC thioredoxin motif of PDI is mutated to CXXA, favoring persistent mixed disulfide crosslinking with substrates (3). Indeed, proAVP interacted much more strongly with PDI-C56A when compared with WT PDI (Figure 8A). PDI-C56A and proAVP also formed higher-molecular-weight complexes including 2 distinct bands (Figure 8B) at approximately $80 \mathrm{kDa}$, corresponding to the cumulative molecular masses of 1 PDI (60 kDa) plus 1 proAVP $(20 \mathrm{kDa})$, and at approximately $100 \mathrm{kDa}$, corresponding to the cumulative molecular masses of 1 PDI plus a proAVP dimer. Both forms were sensitive to reducing conditions, suggesting the involvement of intermolecular disulfide bonds in complex formation. Indeed, knockdown of endogenous PDI in WT cells nearly abolished WT proAVP ubiquitination (Figure 8C) and stabilized proAVP (Figure $8 \mathrm{D})$, pointing to a possible role of PDI in proAVP degradation. Hence, we conclude that PDI forms a direct disulfide bond link with proAVP, rather than an association via an indirect interaction, and that PDI is required for proAVP ERAD, presumably by mediating the reduction and unfolding of proAVP for retrotranslocation.

Enzymatic activity of PDI is required for ERAD deficiency-triggered proAVP aggregation. Last, we asked whether PDI is required for proAVP aggregation in the absence of Sel1L-Hrd1 ERAD. To this end, we perturbed PDI function in ERAD-deficient cells using 2 complementary approaches: ectopic expression of PDIC56A and siRNA knockdown of endogenous PDI. While ERAD deficiency caused proAVP aggregation (middle panel, Figure 9A), ectopic expression of the PDI trap mutant in ERAD-deficient cells sequestered proAVP predominantly in lower-molecular-weight binary complexes (right panel), thereby limiting the ability of PDI-trapped proAVP to form larger protein complexes. Quantification of the abundance of proAVP and proAVP-containing protein complexes in each fraction is shown in Supplemental Figure 6. Furthermore, while having little effect in WT cells, knockdown of PDI attenuated the formation of disulfide bond-mediated high- 
A
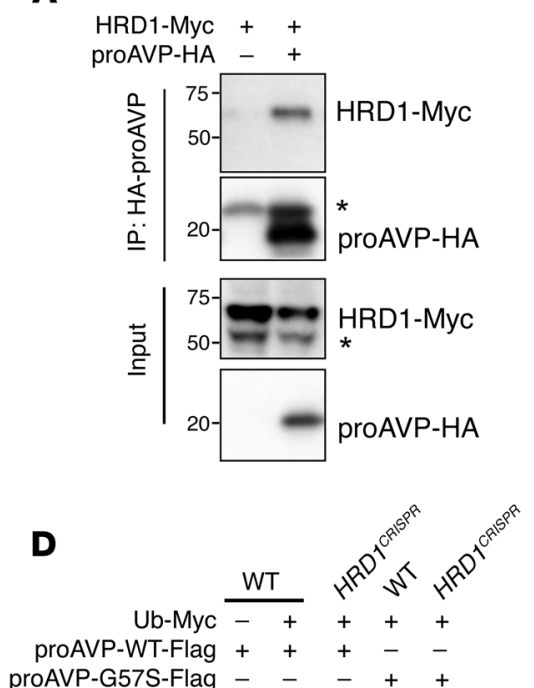

proAVP-G57S-Flag $-{ }_{-}+{ }_{-}+$
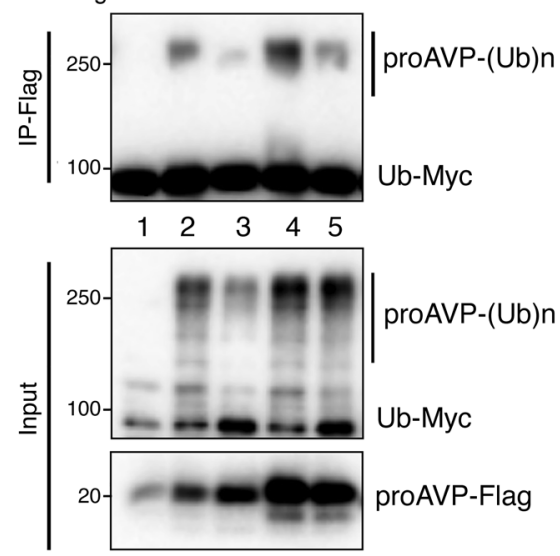

$\mathbf{F}$
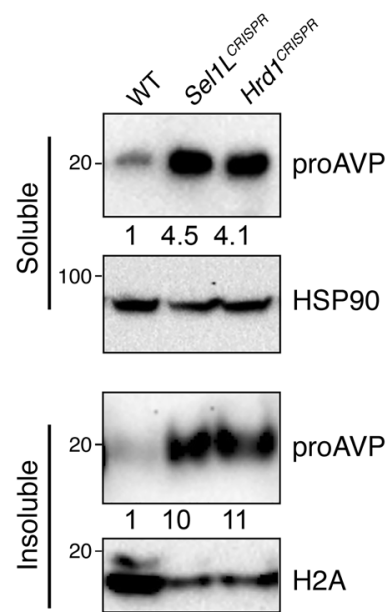

B

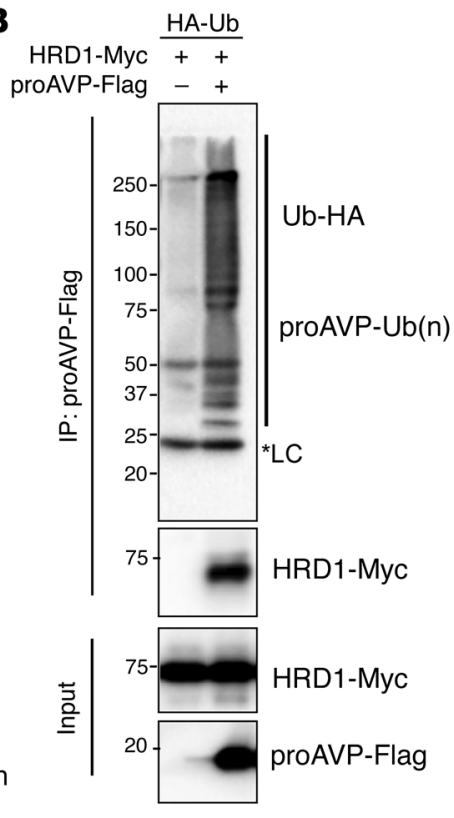

E
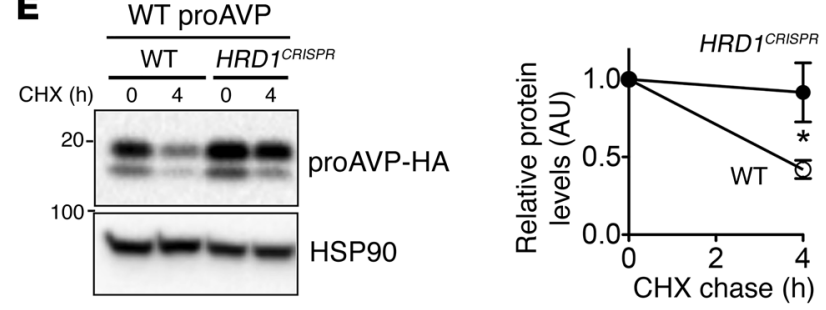

G
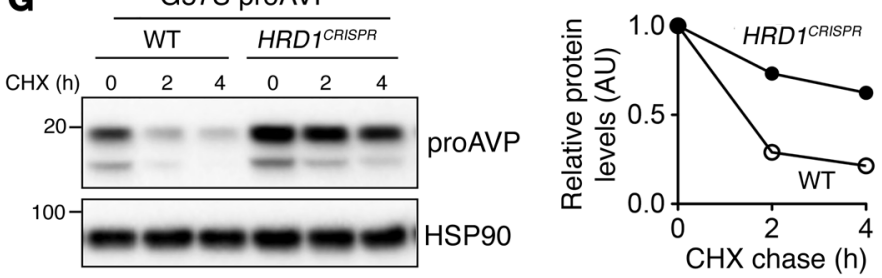

H

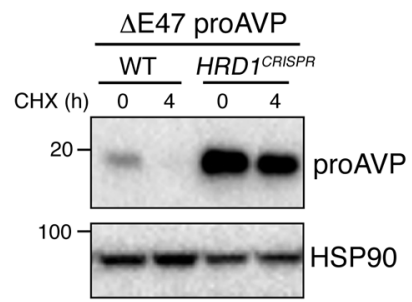

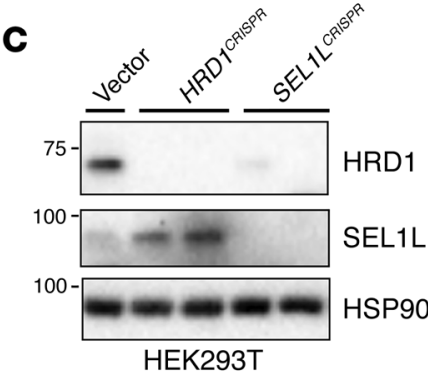

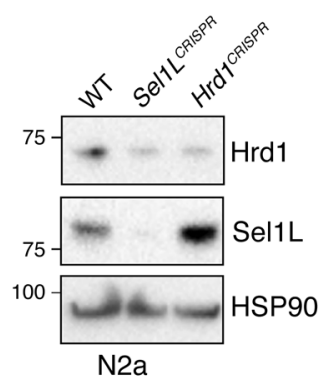

EL1L SP90

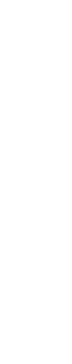



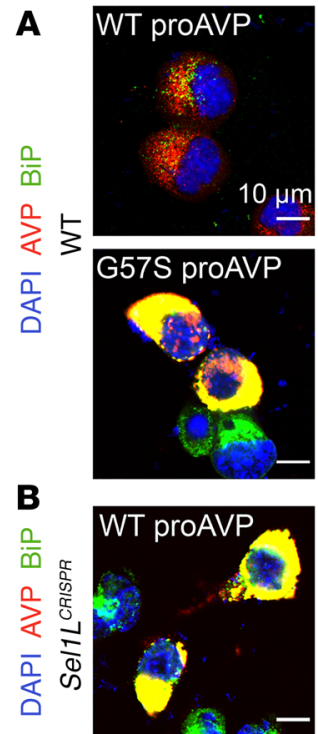

E

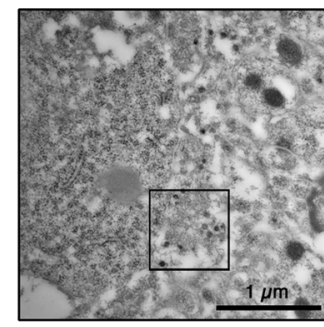

Sel1 $L^{|f| t|i|}$

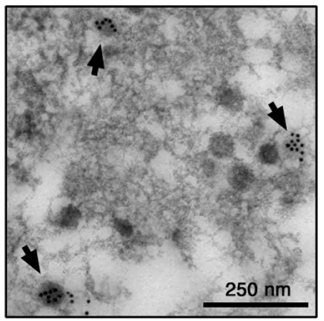

WT
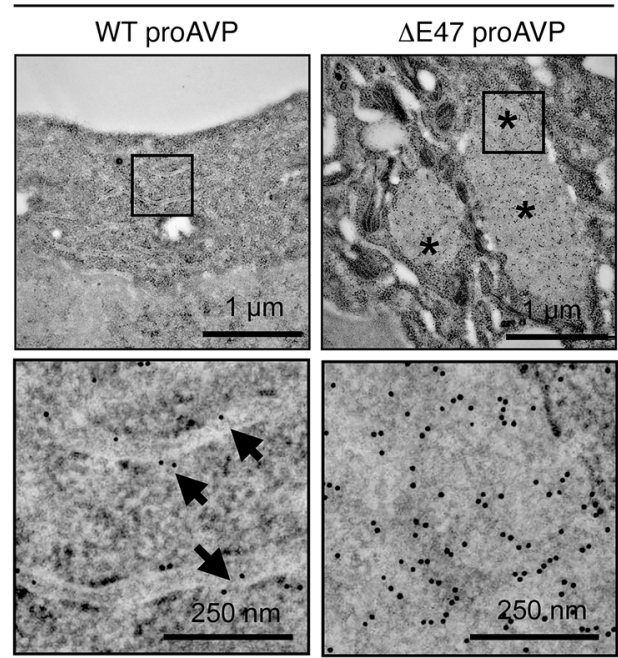

$\mathbf{F}$

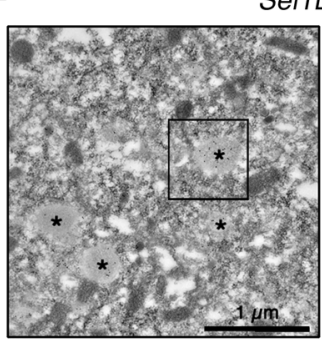

D
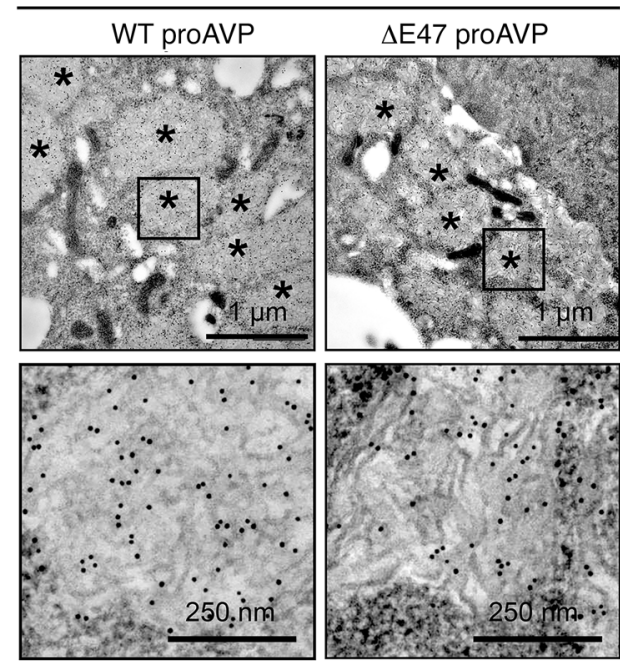

G
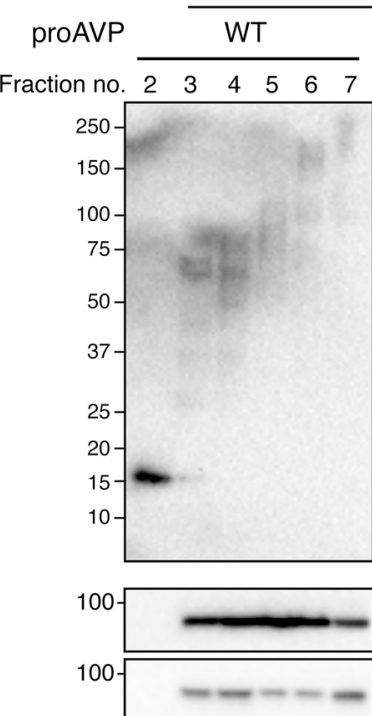

WT
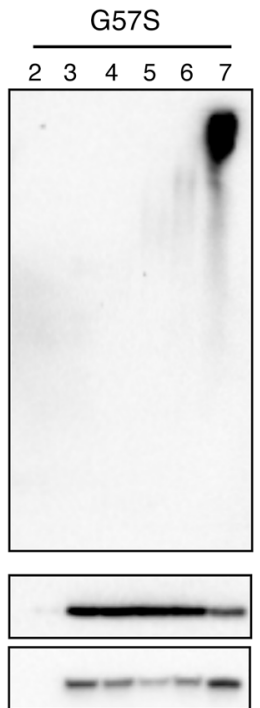

\begin{tabular}{llllll}
\multicolumn{4}{l}{$W T+G 57 S$} \\
\hline 2 & 3 & 4 & 5 & 6 & 7
\end{tabular}

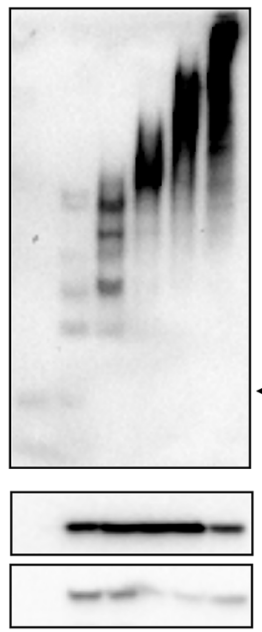

Blot: proAVP

H
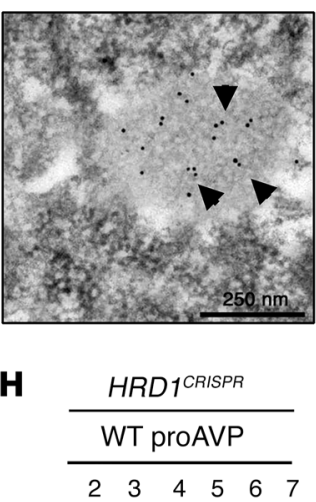

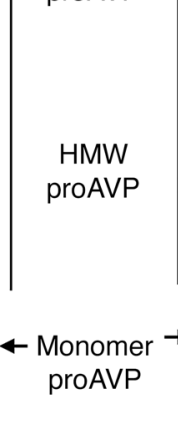

Calnexin

HSP90
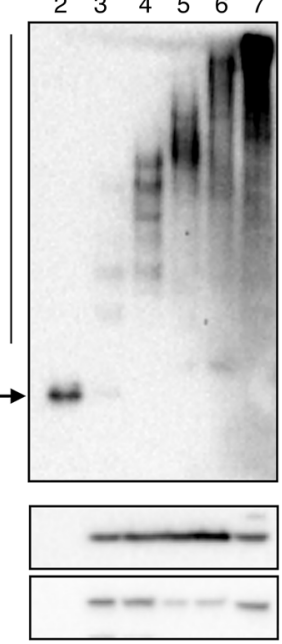

Figure 6. The majority of proAVP proteins form aggregates in the absence of SEL1L-HRD1 ERAD. (A and B) Representative immunofluorescence staining of WT and G57S-mutant proAVP and endogenous BiP in transfected WT (A) and Sel1L-deficient N2a cells (B). (C and D) Immunogold (proAVP, anti-NPII) coupled with TEM analysis of WT (C) or Sel1L-deficient (D) N2a cells expressing WT or $\triangle E 47$ human disease mutant proAVP. Higher-magnification images are shown below. Arrows indicate proAVP in ER sheets; asterisks mark fibrillar proAVP aggregates. (E and F) Immunogold labeling and TEM analysis of endogenous proAVP in the PVN of 10-week-old mice, with higher-magnification images shown on the right. Arrows indicate proAVP-containing dense secretory granules, and asterisks mark aggregates. $n=2$ male mice each. $(\mathbf{G}$ and $\mathbf{H})$ Sucrose fractionation followed by Western blot analyses of the proAVP protein complex in (G) WT HEK293T cells transfected with WT, G57S proAVP, or both at a ratio of 1:1, or (H) HRD1-deficient HEK293T cells transfected with WT proAVP. Fractions were collected from top (no. 1) to bottom (no. 6) and the redissolved pellet as fraction no. 7, followed by Western blot analysis under nonreducing conditions. HSP9O and calnexin represent cytosolic and ER protein controls, respectively. HMW, high-molecular-weight complexes. Data shown are representative of at least 2 independent experiments. 


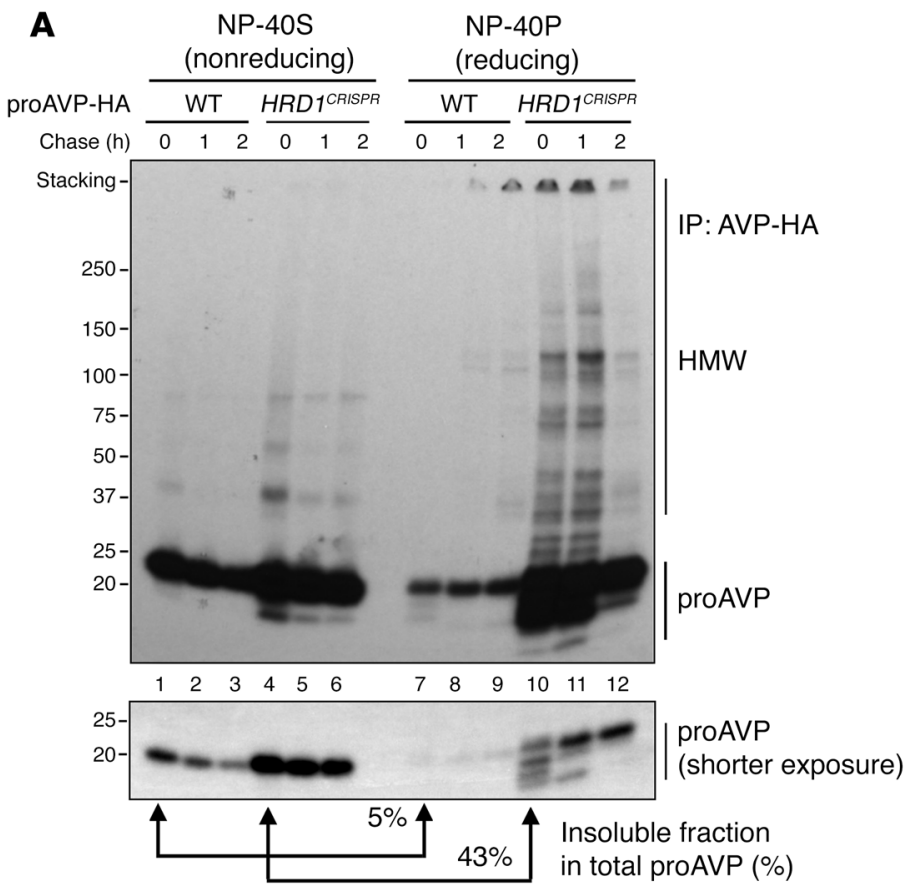

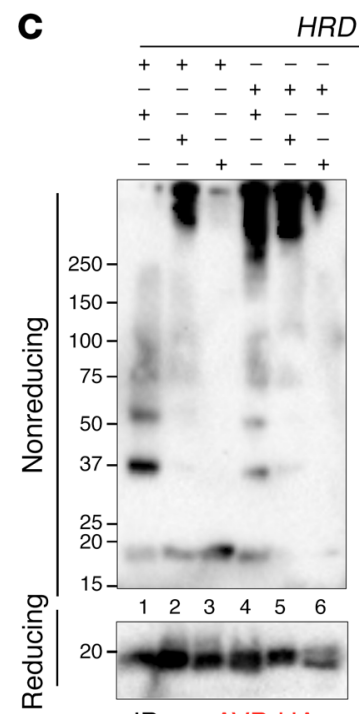

IB: proAVP-HA $+++-\ldots$ - proAVP-WT-HA

- + + + + proAVP-G57S-HA

$+-\ldots+-$ - proAVP-WT-Flag

++-+- proAVP-WT-Flag-KDEL

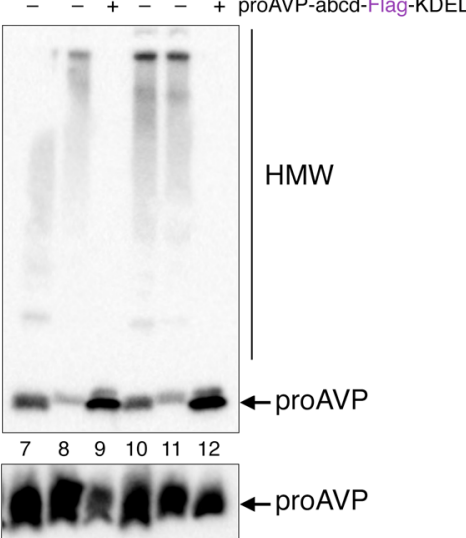

IB: proAVP-Flag

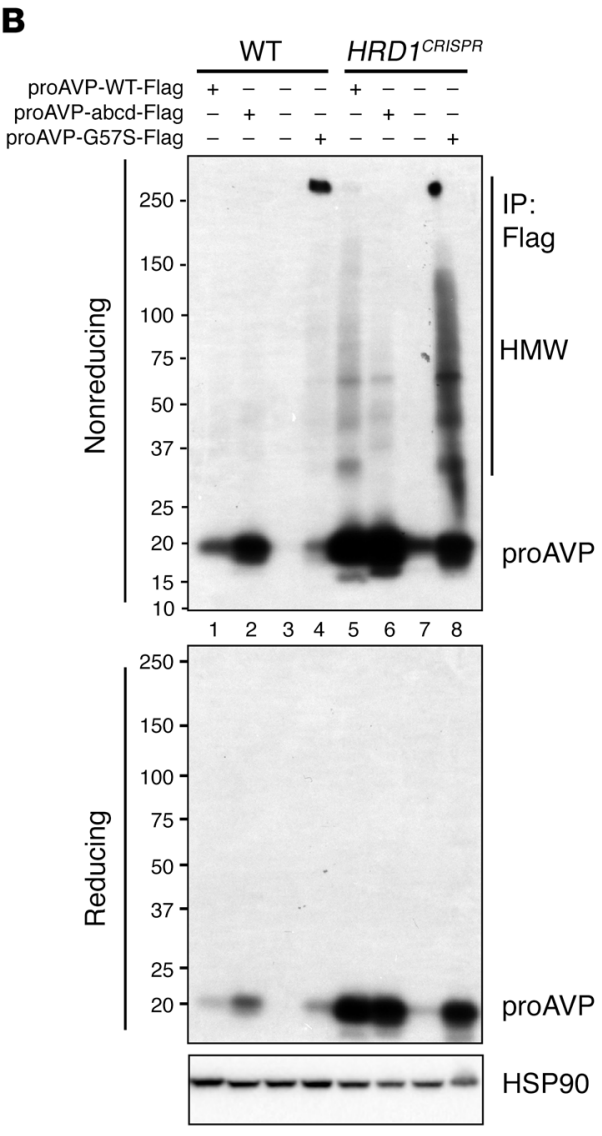

B

Figure 7. SEL1L-HRD1 ERAD deficiency causes proAVP aggregation in a disulfide bond-dependent manner. (A) ${ }^{35} \mathrm{~S}$ pulse-chase (for 15 min) analysis of WT proAVP distribution in WT and HRD1-deficient HEK293T cells. NP-40-soluble (NP-40S) and redissolved insoluble (NP-40P) fractions were immunoprecipitated with anti-HA agarose beads and separated on SDS-PAGE gels under nonreducing (NP-40S) or reducing (NP-40P) conditions, followed by autoradiography. Shorter exposure is shown below. (B) ${ }^{35} \mathrm{~S}$ pulse-labeling and immunoprecipitation analyses of oligomerization of WT or cysteine-less (abcd, Cys to Ser) mutant proAVP in WT or HRD1-deficient HEK293T cells. NP-40-soluble fractions were separated on SDS-PACE gels under nonreducing or reducing (lower) conditions. HSP9O was blotted as a loading control from total cell lysates. (C) Western blot analysis under both nonreducing and reducing conditions of proAVP aggregation in HRD1-deficient HEK293T cells transfected with a different combination of HA- and Flag-tagged proAVP constructs. abcd, cysteine-less proAVP; KDEL, ER retention signal. Data are representative of at least 2 independent experiments.

molecular-weight complexes and aggregates in both SEL1L- and HRD1-deficient cells (Figure 9B). Taken together, we conclude that in ERAD-deficient cells, PDI redox activity is required for the formation of proAVP aggregates or polymers.

\section{Discussion}

Our data show that mammalian SEL1L-HRD1 ERAD is responsible for the clearance of misfolded proAVP in the ER and thereby prevents propagation of this misfolding to additional proAVP molecules via aberrant disulfide bonds (Figure 9C). In the absence of ERAD, misfolded molecules with highly reactive cysteine thiols accumulate and participate in inappropriate intermolecular disulfide-bonded aggregates, a process promoted by the enzymatic activity of PDI (Figure 9C). Mice with Sel1L deficiency due to either acute global KO or to selective deletion in AVP neurons phenocopy central diabetes insipidus in humans $(6,7,45)$. These findings in 2 
A
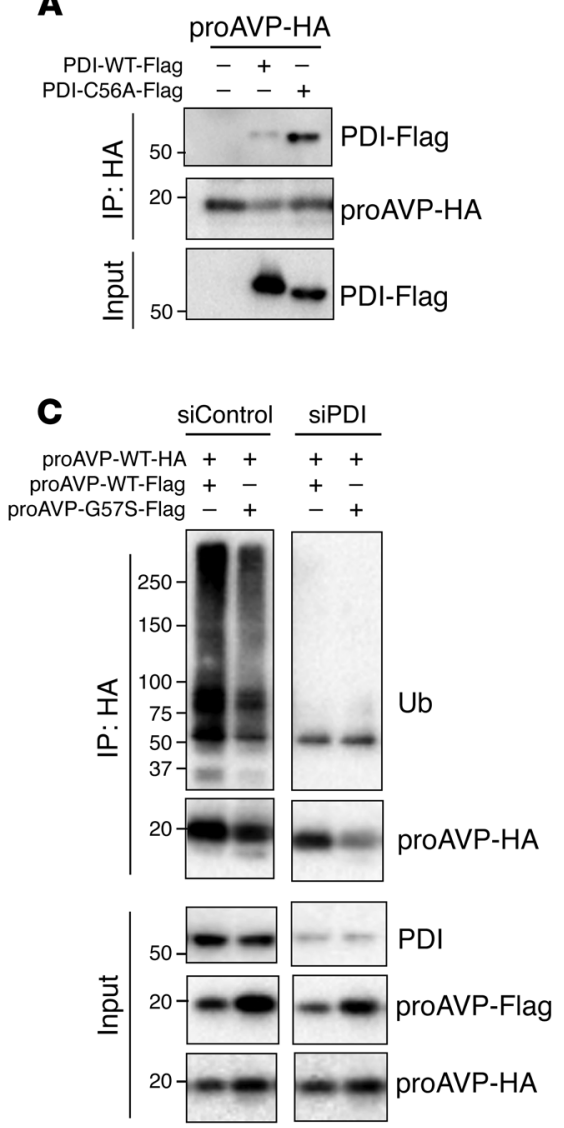

B
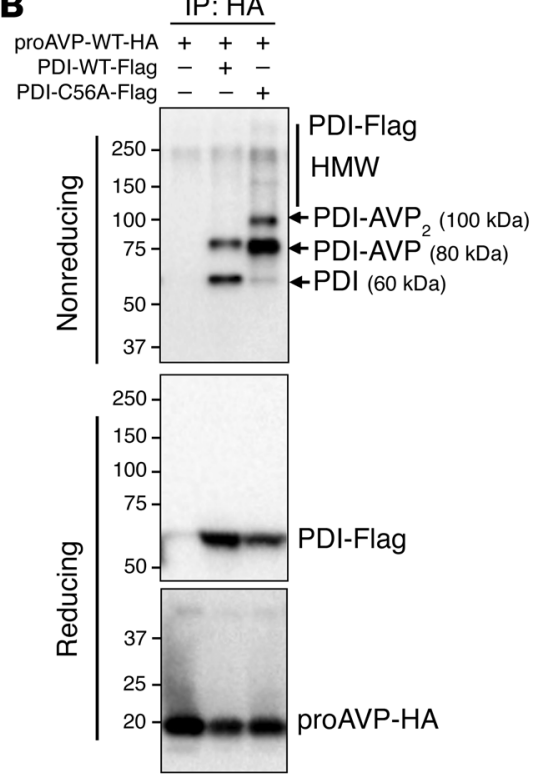

DT proAVP-HA siControl siPDI

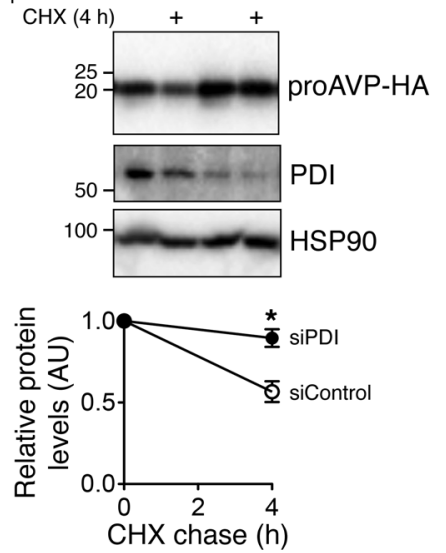

Figure 8. Enzymatic activity of $P D I$ is required for proAVP degradation. (A) Western blot analysis of immunoprecipitates of HA-agarose in proAVP- and/ or PDI-transfected HEK293T cells, showing the interaction between PDI and proAVP. (B) Western blot analysis of proAVP-PDI intermediates in HEK293T cells transfected with WT proAVP plus WT or PDIC56A trap mutant under nonreducing or reducing conditions. (C) Western blot analysis of immunoprecipitates of HA-agarose in proAVP-transfected HEK293T cells, with or without PDI knockdown (siPDI). Two panels were from the same experiment at the same exposure time, with the irrelevant lanes in the middle cut off. (D) Western blot analysis and quantification of WT proAVP protein turnover in HEK293T cells, with or without siPDI. Values represent the mean \pm SEM. ${ }^{*} P<0.05$, by Student's $t$ test. Data shown are representative of at least 2 independent experiments. independent mouse models demonstrate both the significance of ERAD in normal physiology and the potential significance of this pathway in human disease pathogenesis $(11,12)$. Whether this finding is applicable more broadly to other peptide prohormones with similar misfolding behavior remains an open question.

ERAD function and physiological relevance have long been thought to be intimately linked to ER stress and the UPR. Thus far, studies of ERAD in a cell type-specific context lag behind those of the UPR, but evidence published to date suggests that the physiological role of Sel1L-Hrd1 ERAD can be UPR independent, depending on the specific substrate(s) found in specific cell types (20, 21, 25-27, 46-48). In this study, we showed that ER retention of proAVP occurs early and coincides with the initiation of diabetes insipidus in Sel1L-deficient mice. Indeed, neuronal cell death or loss in AVP neurons is undetectable in young ERAD-deficient mice, even when central diabetes insipidus is already present. Our model is reminiscent of the diabetes insipidus mouse models carrying human proAVP mutations, in which AVP neuronal cell death is not observed at the time of disease onset and thus is not thought to play a role in disease initiation $(9,49,50)$. The notion that
ERAD deficiency is not necessarily associated with elevated cell death is further supported by recent reports of ERAD-deficient adipocytes $(25,26)$, B cells (27), and colonic epithelium (21). We speculate that cells with compromised ERAD function may adapt in a number of ways including autophagy, reprogramming of BiP or other ER chaperone expression, and/or sequestering of misfolded proteins into "nontoxic" amyloid aggregates (51) in order to reset the parameters of ER homeostasis and thereby allow cells to survive for an extended period of time. Hence, we conclude that ERAD may be causally involved in disease initiation in a substratespecific manner. This conclusion underscores the importance of an improved understanding of cell type-specific ERAD function in the pathogenesis of many human diseases.

While ERAD has largely been studied as a mechanism for clearance of misfolded model substrates (11, 12), our findings uncover what we believe to be a novel role of ERAD in the folding of at least a subset of prohormones in endocrine cells under physiological and pathological contexts. Endocrine cells such as AVP neurons are committed and specifically adapted to make specialized prohormones at high levels; however, such high levels may make 
A

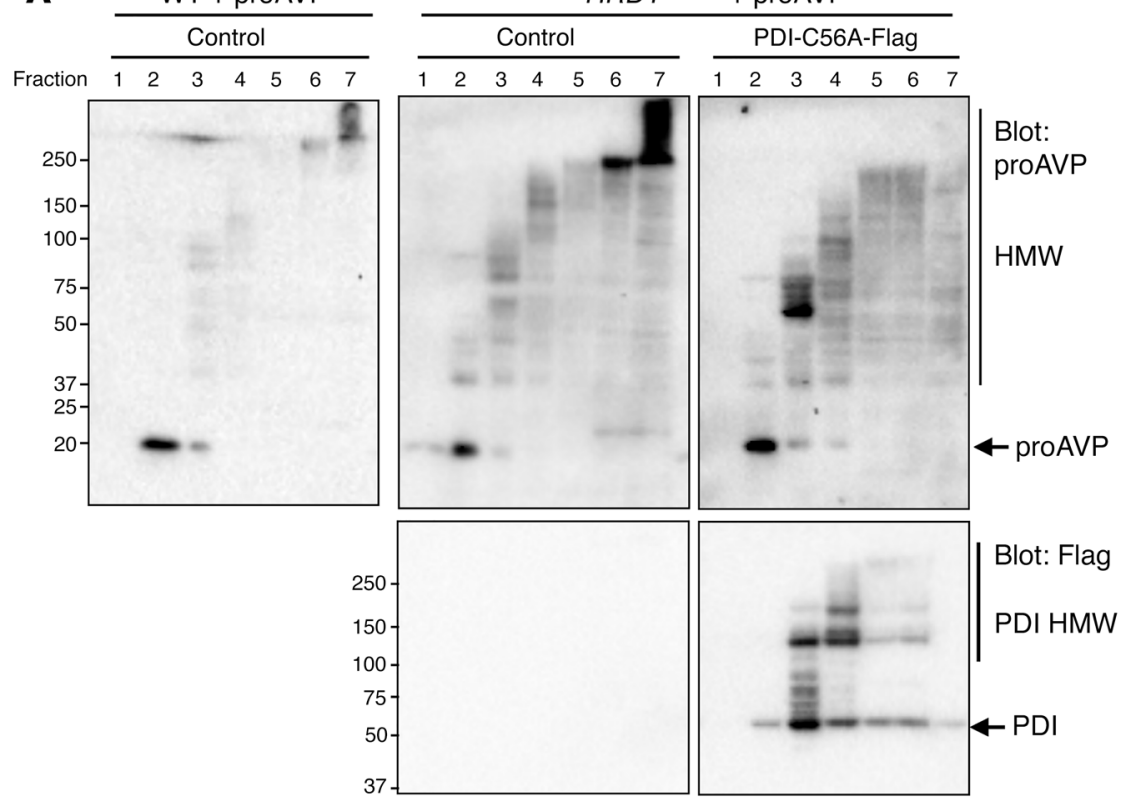

B
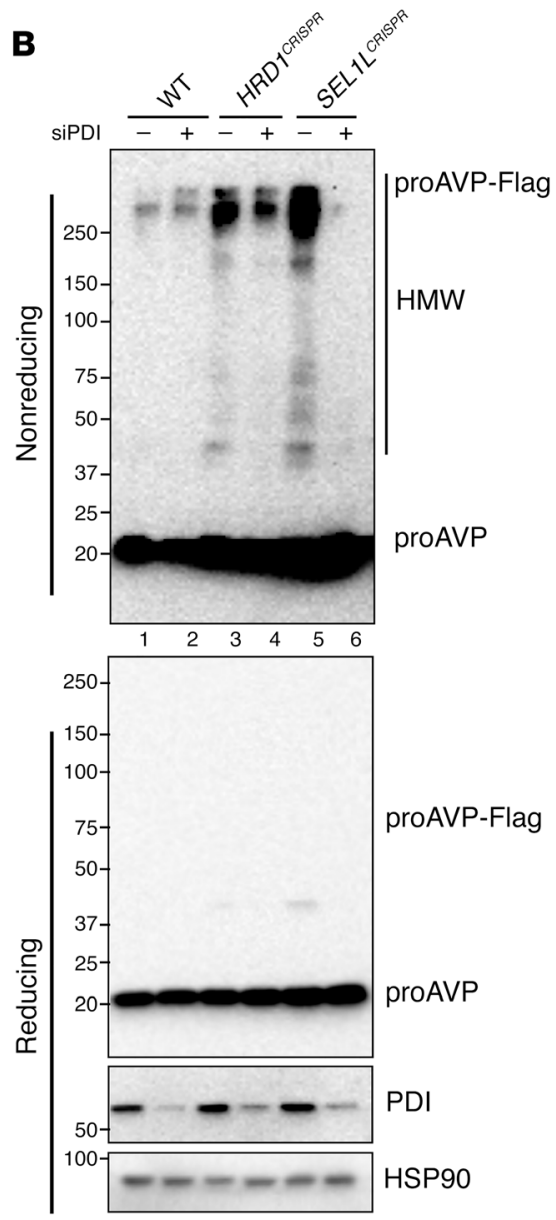

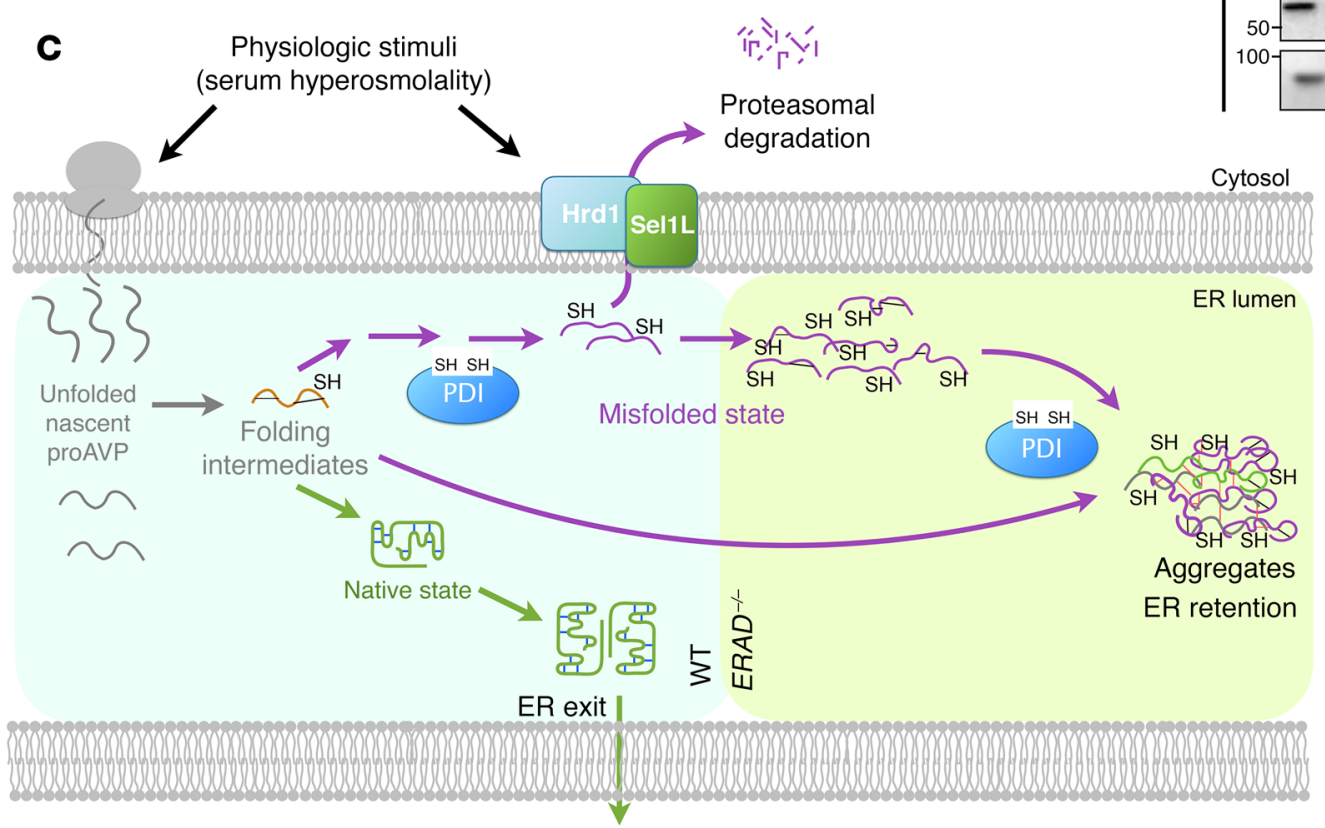

Figure 9. Enzymatic activity of PDI is required for ERAD deficiency-triggered proAVP aggregation and the model. (A) Sucrose fractionation followed by Western blot analyses of the proAVP protein complex (using the proAVP antibody PS41) in HRD1-deficient HEK293T cells, with or without a PDI-C56A trap mutant. Quantification of lane intensity of the proAVP Western blot is shown in Supplemental Figure 6. (B) Western blot analysis of proAVP aggregation under both nonreducing and reducing conditions in WT and PDI-deficient HEK293T cells transfected with control siRNA or siPDI, respectively. Data are representative of at least 2 independent experiments. (C) Model for the role of Sel1L-Hrd1 ERAD in proAVP conformational maturation in the ER. Physiological stress such as hyperosmolality induces the expression of proAVP and Sel1L-Hrd1 ERAD, which in turn clears misfolded proAVP - a critical process for the formation of natively folded dimers. Our data suggest that Sel1L-Hrd1 ERAD plays an important role in promoting a safe environment for nascent proteins, allowing them to reach native conformation without the distraction of aggregation, a process that may require PDI. 
the conformational maturation process more susceptible to generating errant products. Intriguingly, our data suggest that ERAD activity in AVP neurons is a critical part of the response to serum hyperosmolality, coordinated with AVP production. Thus, serum osmolality represents a physiological cue that tightly links ERAD to proAVP maturation for the control of systemic water balance. How ERAD is regulated by serum hyperosmolality is an interesting open question. As IRE1a of the UPR is known to integrate extracellular cues to regulate ERAD gene expression in yeast and mammals (15, $21,52-54)$, we speculate that IRE1 $\alpha$ of the UPR may link hyperosmolality to Sel1L-Hrd1 ERAD in the AVP neurons via its downstream effector transcription factor XBP1s. XBP1s may directly regulate the transcription of Sel1L-Hrd1 ERAD components, as we have previously described in enterocytes (21). If true, this model would predict an intimate crosstalk between two ER quality-control machineries (IRE1 $\alpha$ of the UPR and Sel1L-Hrd1 of ERAD) in the control of prohormone maturation and systemic water balance. Future studies are required to directly test this hypothesis.

Recent studies in mouse models have suggested varying effects of ERAD on the fate of different substrates (11). For example, IRE1 $\alpha$, which is a Sel1L-Hrd1 ERAD substrate in many cell types (20), accumulates in the ER but remains functional to sense misfolded ER proteins in ERAD-deficient cells. The pre-B cell receptor, a Sel1L-Hrd1 ERAD substrate in developing $B$ cells, accumulates in the ER and leads to an increased level of functional proteins at the cell surface of ERAD-deficient cells (27). In both cases, ERAD deficiency leads to a gain-of-function phenotype mimicking the effect of substrate overexpression. By contrast, this study reveals a totally different fate for an ERAD substrate; specifically, ERAD deficiency results in the disruption of proAVP conformational maturation in the ER, generating a loss-of-function proAVP phenotype. The differences in substrate fate are probably dictated by several substrate-specific factors including, but not limited to, the chemical concentration of the substrate protein, the predisposition for oligomerization of secretory proteins in the ER, and the specific location of exposed or buried cysteines within the substrate. Oligomerization or selfassociation may occur concurrently with the folding of individual monomers, which makes the folding status of each of the individual monomeric partners a contributor to the fate of the prohormone. This may explain why, in ERAD-deficient cells, there is a failure to undergo conformational maturation for the majority of proAVP. In addition to being an ERAD complex for proAVP turnover, the Sel1L-Hrd1 protein complex may act as a holdase in the conformational maturation of proAVP, the importance of which requires additional studies. Thus, the physiological relevance and significance of Sel1L-Hrd1 ERAD needs to be dissected carefully in a cell type- and substrate-specific manner.

Another novelty of this study, we believe, is the importance of PDI in aggregate formation in cells with impaired ERAD activity. Studies have suggested that PDI subserves a pro-folding role in WT cells $(30,55)$, and more recently, studies have demonstrated that PDI, but not other members of the PDI family (ERp57 and ERdj5), may also participate in ERAD by feeding reduced protein substrates (e.g., Akita proinsulin and thyroglobulin) to the ERAD machinery $(3,33,56)$. Our data expand these views by showing that, in cells with impaired ERAD function, PDI may promote, rather than prevent, the formation of proAVP aggregates or polymers. Given our own data and the current literature $(3,33,56)$, we propose 2 possible modes of action for PDI in the context of proAVP ERAD, as illustrated in Figure 9C. PDI may act to reduce and unfold proAVP targeted for ERAD, which may result in the generation of unfolded substrates with highly reactive cysteine thiols. In ERAD-deficient cells, these unfolded substrates have no place to go other than to engage in a nonproductive pathway of protein aggregation via aberrant disulfide bonds. Alternatively, or additionally, PDI may be directly involved in aggregate formation by catalyzing the formation or isomerization of intermolecular disulfide bonds among proAVP proteins in the aggregates. Although lacking substantial evidence, we speculate that, as suggested for certain amyloid fibrils (57), the formation of proAVP polymers might actually be less cytotoxic as a means of sequestering the misfolded proteins, thereby allowing the secretory pathway to continue with only minimal perturbation.

The most striking feature of this study is the profound effect of SEL1L-HRD1 ERAD deficiency on proAVP conformational maturation that leads to disease development. While it is not surprising that ERAD degrades a fraction of newly synthesized unstable proAVP, our data reveal a different role for ERAD: that is, ERAD provides a safe environment for nascent proteins, allowing them to reach proper folding conformation without the distraction of aggregation. Thus, the findings from this study will provide insights into the pathogenesis of various human diseases associated with protein aggregation. We propose that boosting ERAD function and/or increasing the efficacy of targeting substrates to ERAD machinery may be of therapeutic value in helping to improve prohormone conformational maturation in the ER. Hence, this study may open a new line of research into ERAD and prohormone biology as well as disease pathogenesis associated with defects in prohormone maturation and protein aggregation.

\section{Methods}

Mice. The Sel1L $L^{f l f l}$ and tamoxifen-inducible Sel1 $L^{E R C r e}$ mice were previously described (20). The Sel1L $L^{f / f l}$ mice backcrossed with C57BL/6 mice at least 5 times were crossed with mice expressing AVP promoter-driven Cre on the C57BL/6J background (JAX 023530, B6.Cg-Avp $p^{t m 1.1 /(\text { cre)Hze } / J ; ~ T h e ~ J a c k s o n ~ L a b o r a t o r y) ~ t o ~ g e n e r a t e ~ A V P ~ n e u-~}$ ron-specific Sel1L-deficient mice (Sel1L ${ }^{\text {AVP }}$ ) and control littermates. Mice were fed a low-fat diet (13\% fat, 67\% carbohydrate, and $20 \%$ protein, 2914; Harlan Teklad). Metabolic cage analysis was performed with a comprehensive laboratory animal monitoring system (CLAMS) (Columbus Instruments) with a 1-day acclimation period, followed by 2 days of measurements. Animals were housed in a 12-hour light/12-hour dark cycle and under controlled temperatures $\left(20^{\circ} \mathrm{C}-22^{\circ} \mathrm{C}\right)$ in the mouse facility.

Immunofluorescence staining and immunohistochemistry. Freefloating brain sections were permeabilized by $0.3 \%$ Triton X-100 for 10 minutes at room temperature and then incubated in blocking solution (1\% donkey serum and $0.03 \%$ Triton $\mathrm{X}-100$ in $0.05 \mathrm{M}$ potassium phosphate-buffered saline [K-PBS]) for 30 minutes at room temperature. After blocking, free-floating brain sections were simultaneously incubated overnight with primary antibodies at $4^{\circ} \mathrm{C}$ and, following 3 washes with K-PBST (0.03\% Triton X-100 in $0.05 \mathrm{M}$ K-PBS), were incubated with secondary antibodies for 2 hours at room temperature. 
Brain sections were then mounted on gelatin-coated slides (SLD01CS; Southern Biotech). Counterstaining and mounting were performed with mounting medium containing DAPI (Vector H-1200) and a Fisherfinest Premium Coverslip (12-548-5P; Thermo Fisher Scientific). For immunostaining of N2a cells, a 12-mm-diameter cover glass (72230-01; Electron Microscopy Sciences) was coated with $0.1 \%$ polyL-lysine solution (P8920; Sigma-Aldrich). Cells $\left(1 \times 10^{4}\right)$ were placed on a coated cover glass in a 24 -well plate and then transfected with proAVP constructs using Lipofectamine 2000 (Thermo Fisher Scientific). After transfection ( 24 hours later), cells were fixed by $4 \%$ formaldehyde (89370-094; VWR) for 10 minutes at room temperature and then washed with K-PBS and permeabilized using 0.3\% Triton X-100 for 10 minutes at room temperature, followed by incubation in blocking solution. Cells were stained as described above. For immunofluorescence staining of paraffin-embedded kidney sections, sections were rehydrated and boiled in $1 \mathrm{mM}$ EDTA for antigen retrieval, followed by antibody labeling as described above.

Transmission electron microscopy. Cultured N2a cells were transfected with various constructs. After transfection (48 hours after), cells were fixed in medium containing 3\% formaldehyde and $0.2 \%$ glutaraldehyde and incubated for 2 hours at room temperature, followed by overnight incubation at $4^{\circ} \mathrm{C}$. Cells were collected by scraping and centrifugation for 10 minutes at 2,500 $\mathrm{g}$, washed 3 times with PBS, incubated with $50 \mathrm{mM} \mathrm{NH}_{4} \mathrm{Cl}$ in PBS for 30 minutes, washed 3 times in PBS, dehydrated at $4^{\circ} \mathrm{C}$ in serially diluted methanol, and infiltrated with LR-Gold resin according to the manufacturer's instructions (London Resin). Polymerization was performed for 1 day at $-10^{\circ} \mathrm{C}$ under UV light. For Immunogold labeling, sections of approximately $70-\mathrm{nm}$ thickness were collected on carbon-coated Formvar-Ni grids incubated for 15 minutes with PBS containing 2\% BSA and 0.1\% Tween-20 for 2 hours at room temperature with a homemade polyclonal rabbit anti-proAVP antiserum (raised against residues 28-145, i.e., the C-terminal portion of NPII and the GP) diluted in the same buffer, washed 5 times for 5 minutes in a drop of PBS, and incubated for 2 hours with colloidal gold-conjugated secondary goat anti-rabbit Ig antibody (BioCell) in PBS, 2\% BSA, and 0.1\% Tween-20. Grids were washed 5 times for 5 minutes in PBS and then 5 times in $\mathrm{H}_{2} \mathrm{O}$. Sections were stained for 15 minutes with $2 \%$ uranylacetate and 2 minutes with lead citrate (Reynold's solution) and then finally viewed with a Philips CM 100 electron microscope (5). For immunogold labeling of brain tissue, sections were stained using mouse monoclonal anti-proAVP antibody PS41 (diluted 1:200) overnight at $4^{\circ} \mathrm{C}$ and gold-conjugated secondary goat anti-mouse Ig antibody (BioCell).

Antibodies for Western blotting and immunostaining. The following antibodies were used for Western blotting and immunostaining: Sel1L (rabbit, 1:2,000 for Western blotting; ab78298; Abcam); BiP/ GRP78 (goat, 1:1,000 for Western blotting and 1:200 for immunostaining; sc-1051; Santa Cruz Biotechnology Inc.); AQP2 (goat, 1:200 for immunostaining; sc-9882; Santa Cruz Biotechnology Inc.); HSP90 (rabbit, 1:6,000 for Western blotting; sc-7947; Santa Cruz Biotechnology Inc.); c-Fos (rabbit, 1:200 for immunostaining; sc-52; Santa Cruz Biotechnology Inc.); Flag (mouse, 1:200 for immunostaining; F-1804; Sigma-Aldrich); PDIA1 (rabbit, 1:8,000 for Western blotting; SPA-890; Assay Design or Enzo); Flag (mouse, 1:8,000 for Western blotting; F1804; Sigma-Aldrich); and HA (mouse, 1:5,000 for Western blotting; H9658; Sigma-Aldrich). Anti-Hrd1 antibody (rabbit, 1:300 for immunostaining) was provided by Richard Wojcikiewicz
(SUNY Upstate Medical University, Syracuse, New York, USA). AntiNPII (mouse, 1:200 for immunostaining of proAVP and mature NPII, PS41) and anti-AVP (rabbit, 1:200 for immunostaining of proAVP and mature AVP, VA4) were provided by Harold Gainer (National Institute of Neurological Disorders and Stroke, NIH, Bethesda, Maryland, USA) (40, 58). Anti-H2A (rabbit, 1:10,000 for Western blotting) was a gift of Yihong Ye (National Institute of Diabetes and Digestive and Kidney Diseases, NIH). The secondary antibodies used for Western blotting were: goat anti-rabbit IgG HRP and goat anti-mouse IgG HRP (1:5,000; both from Bio-Rad). Donkey anti-goat IgG was obtained from Jackson ImmunoResearch Laboratories. The secondary antibodies used for fluorescence immunostaining (all 1:500) were: anti-mouse IgG Cy3 and FITC; anti-rabbit IgG Alexa Fluor 488, Alexa Fluor 594, and Alexa Fluor 647; and anti-goat IgG Alexa Fluor 594 and Alexa Fluor 680 (Jackson ImmunoResearch). See the complete unedited blots in the supplemental material.

CRISPR/Cas9-based gene editing. Generation of HRD1-deficient HEK293T cells was previously described (20). Single-guide RNA (sgRNA) oligonucleotides were designed for human HRD1 (5'-GGACAAAGGCCTGGATGTAC). To generate Sel1L- and Hrd1-deficient mouse N2a cells, sgRNA oligonucleotide for mouse Sel1L (GCCAGCAACTACTTTGCCCG) or mouse Hrd1 (ATCCATGCGGCATGTCGGGC) was inserted into lentiCRISPR v2 (plasmid 52961; Addgene). We used third-generation lentiviral packaging plasmids (RRE [gag/ pol], VSV-G and REV) to deliver CRISPR constructs into the cells. Cells were cultured 24 hours after infection in medium containing 2 $\mu \mathrm{g} / \mathrm{ml}$ puromycin for 24 hours and then in normal growth media.

Additional materials and methods are described in the Supplemental materials.

Statistics. Results are expressed as the mean \pm SEM unless otherwise stated. Comparisons between groups were made by unpaired, 2-tailed Student's $t$ test, where a $P$ value of less than 0.05 was considered statistically significant. All experiments were repeated at least 2 to 3 times or performed with several independent biological samples, and representative data are shown.

Study approval. All experiments performed with mice were in compliance with the IACUCs of Cornell University (approval 20070051) and the University of Michigan (approval PRO00006888).

\section{Author contributions}

GS and DRMS designed the experiments and performed most of the experiments; GHK taught DRMS the brain-related technique and performed some of the experiments; SS performed metabolic cage studies and initial studies; CPB, NB, JR, and MS performed Immunogold transmission electron microscopy (TEM) experiments and provided key reagents and discussions; QL provided key reagents; PA offered suggestions and edited the manuscript; GS, GHK, and DRMS wrote the figure legends and methods; LQ designed the experiments, supervised the work, and wrote the manuscript; all authors reviewed, edited, and approved the manuscript.

\section{Acknowledgments}

We thank Harold Gainer, Ming Liu, Richard Wojcikiewicz, Haoquan Wu, Yihong Ye, Billy Tsai, and Hideki Nishitoh for reagents; David Olson for critical comments on our manuscript; and members of the Qi and Arvan laboratories for comments and technical assistance. This work was supported by grants from the NIH 
(R01DK48280, to PA); NIH (R01DK111174, to PA and LQ); the University of Michigan Protein Folding Diseases Initiative (to LQ and PA); the Swiss National Science Foundation (31003A-162643, to MS); the American Diabetes Association (ADA) (1-12-CD-04) and the NIH (R01GM113188 and R01DK105393, to LQ). GHK is supported by an ADA Postdoctoral Fellowship grant (1-17-PDF142). SS is an International Student Research Fellow of the Howard Hughes Medical Institute (59107338). LQ is the recipient of the Junior Faculty and Career Development Awards from the ADA.
Address correspondence to: Ling Qi, 1000 Wall Street, University of Michigan Medical School, Ann Arbor, Michigan 48105, USA. Phone: 734.936.4720; Email: lingq@med.umich.edu.

DRMS's present address is: Yale University School of Medicine, New Haven, Connecticut, USA.

SS's present address is: Department of Pharmacology, University of Texas Southwestern Medical Center, Dallas, Texas, USA.
1. Westphal $\mathrm{CH}$, et al. The neuroendocrine protein $7 \mathrm{~B} 2$ is required for peptide hormone processing in vivo and provides a novel mechanism for pituitary Cushing's disease. Cell.1999;96(5):689-700.

2. Phillips JA. Dominant-negative diabetes insipidus and other endocrinopathies. JClin Invest. 2003;112(11):1641-1643.

3. He K, Cunningham CN, Manickam N, Liu M, Arvan P, Tsai B. PDI reductase acts on Akita mutant proinsulin to initiate retrotranslocation along the Hrd1/Sel1L-p97 axis. Mol Biol Cell. 2015;26(19):3413-3423.

4. Fujiwara TM, Bichet DG. Molecular biology of hereditary diabetes insipidus. J Am Soc Nephrol. 2005;16(10):2836-2846.

5. Birk J, Friberg MA, Prescianotto-Baschong C, Spiess M, Rutishauser J. Dominant pro-vasopressin mutants that cause diabetes insipidus form disulfide-linked fibrillar aggregates in the endoplasmic reticulum. J Cell Sci. 2009;122(Pt 21):3994-4002.

6. Ito M, Jameson JL, Ito M. Molecular basis of autosomal dominant neurohypophyseal diabetes insipidus. Cellular toxicity caused by the accumulation of mutant vasopressin precursors within the endoplasmic reticulum. J Clin Invest. 1997;99(8):1897-1905

7. Ito M, Yu RN, Jameson JL. Mutant vasopressin precursors that cause autosomal dominant neurohypophyseal diabetes insipidus retain dimerization and impair the secretion of wild-type proteins. J Biol Chem. 1999;274(13):9029-9037.

8. Rittig S, et al. Identification of 13 new mutations in the vasopressin-neurophysin II gene in 17 kindreds with familial autosomal dominant neurohypophyseal diabetes insipidus. Am J Hum Genet. 1996;58(1):107-117.

9. Hagiwara D, et al. Arginine vasopressin neuronal loss results from autophagy-associated cell death in a mouse model for familial neurohypophysial diabetes insipidus. Cell Death Dis. 2014;5:e1148.

10. Friberg MA, Spiess M, Rutishauser J. Degradation of wild-type vasopressin precursor and pathogenic mutants by the proteasome. J Biol Chem. 2004;279(19):19441-19447.

11. Qi L, Tsai B, Arvan P. New Insights into the Physiological Role of Endoplasmic Reticulum-Associated Degradation. Trends Cell Biol. 2017;27(6):430-440.

12. Guerriero CJ, Brodsky JL. The delicate balance between secreted protein folding and endoplasmic reticulum-associated degradation in human physiology. Physiol Rev. 2012;92(2):537-576.

13. Hampton RY, Gardner RG, Rine J. Role of $26 \mathrm{~S}$ proteasome and $\mathrm{HRD}$ genes in the degradation of 3-hydroxy-3-methylglutaryl-CoA reductase, an integral endoplasmic reticulum membrane protein. Mol Biol Cell. 1996;7(12):2029-2044.

14. Gardner RG, et al. Endoplasmic reticulum degradation requires lumen to cytosol signaling. Transmembrane control of Hrd1p by Hrd3p. JCell Biol. 2000;151(1):69-82.

15. Christianson JC, et al. Defining human ERAD networks through an integrative mapping strategy. Nat Cell Biol. 2011;14(1):93-105.

16. Christianson JC, Shaler TA, Tyler RE, Kopito RR. OS-9 and GRP94 deliver mutant alpha1-antitrypsin to the Hrd1-SEL1L ubiquitin ligase complex for ERAD. Nat Cell Biol. 2008;10(3):272-282.

17. Mueller B, Lilley BN, Ploegh HL. SEL1L, the homologue of yeast Hrd3p, is involved in protein dislocation from the mammalian ER.JCell Biol. 2006;175(2):261-270.

18. Lilley BN, Ploegh HL. Multiprotein complexes that link dislocation, ubiquitination, and extraction of misfolded proteins from the endoplasmic reticulum membrane. Proc Natl Acad Sci U S A. 2005;102(40):14296-14301.

19. Mueller B, Klemm EJ, Spooner E, Claessen $\mathrm{JH}$, Ploegh HL. SEL1L nucleates a protein complex required for dislocation of misfolded glycoproteins. Proc Natl Acad Sci U S A. 2008;105(34):12325-12330.

20. Sun S, et al. Sel1L is indispensable for mammalian endoplasmic reticulum-associated degradation, endoplasmic reticulum homeostasis, and survival. Proc Natl Acad Sci U S A. 2014;111(5):E582-E591.

21. Sun S, et al. IRE1 $\alpha$ is an endogenous substrate of endoplasmic-reticulum-associated degradation. Nat Cell Biol. 2015;17(12):1546-1555.

22. Vashistha N, Neal SE, Singh A, Carroll SM, Hampton RY. Direct and essential function for Hrd3 in ER-associated degradation. Proc Natl Acad Sci U S A. 2016;113(21):5934-5939.

23. Yagishita N, et al. Essential role of synoviolin in embryogenesis. J Biol Chem. 2005;280(9):7909-7916.

24. Francisco AB, et al. Deficiency of suppressor enhancer Lin12 1 like (SEL1L) in mice leads to systemic endoplasmic reticulum stress and embryonic lethality. J Biol Chem. 2010;285(18):13694-13703.

25. Sha $\mathrm{H}$, et al. The ER-associated degradation adaptor protein Sel1L regulates LPL secretion and lipid metabolism. Cell Metab. 2014;20(3):458-470.

26. Fujita $\mathrm{H}$, et al. The E3 ligase synoviolin controls body weight and mitochondrial biogenesis through negative regulation of PGC-1 1 . EMBO J. 2015;34(8):1042-1055.

27. Ji Y, et al. The Sel1L-Hrd1 endoplasmic reticu- lum-associated degradation complex manages a key checkpoint in B cell development. Cell Rep. 2016;16(10):2630-2640.

28. Feige MJ, Hendershot LM. Disulfide bonds in ER protein folding and homeostasis. Curr Opin Cell Biol. 2011;23(2):167-175.

29. Rutkevich LA, Cohen-Doyle MF, Brockmeier U, Williams DB. Functional relationship between protein disulfide isomerase family members during the oxidative folding of human secretory proteins. Mol Biol Cell. 2010;21(18):3093-3105.

30. Ali Khan H, Mutus B. Protein disulfide isomerase a multifunctional protein with multiple physiological roles. Front Chem. 2014;2:70.

31. Jessop CE, Chakravarthi S, Garbi N, Hämmerling GJ, Lovell S, Bulleid NJ. ERp57 is essential for efficient folding of glycoproteins sharing common structural domains. EMBO J. 2007;26(1):28-40.

32. Irvine AG, et al. Protein disulfide-isomerase interacts with a substrate protein at all stages along its folding pathway. PLOS ONE. 2014;9(1):e82511.

33. Rajpal G, Schuiki I, Liu M, Volchuk A, Arvan P. Action of protein disulfide isomerase on proinsulin exit from endoplasmic reticulum of pancreatic $\beta$-cells. J Biol Chem. 2012;287(1):43-47.

34. Grubb S, Guo L, Fisher EA, Brodsky JL. Protein disulfide isomerases contribute differentially to the endoplasmic reticulum-associated degradation of apolipoprotein B and other substrates. $\mathrm{Mol}$ Biol Cell. 2012;23(4):520-532.

35. Aronson AS, Svenningsen NW. DDAVP test for estimation of renal concentrating capacity in infants and children. Arch Dis Child. 1974;49(8):654-659.

36. Ji LL, Fleming T, Penny ML, Toney GM, Cunningham JT. Effects of water deprivation and rehydration on c-Fos and FosB staining in the rat supraoptic nucleus and lamina terminalis region. Am J Physiol Regul Integr Comp Physiol. 2005;288(1):R311-R321.

37. Luckman SM, Dyball RE, Leng G. Induction of c-fos expression in hypothalamic magnocellular neurons requires synaptic activation and not simply increased spike activity. J Neurosci. 1994;14(8):4825-4830.

38. Qi L, Yang L, Chen H. Detecting and quantitating physiological endoplasmic reticulum stress. Meth Enzymol. 2011;490:137-146.

39. Ben-Barak Y, Russell JT, Whitnall MH, Ozato K, Gainer H. Neurophysin in the hypothalamoneurohypophysial system. I. Production and characterization of monoclonal antibodies. J Neurosci. 1985;5(1):81-97.

40. Whitnall MH, Key S, Ben-Barak Y, Ozato K, 
Gainer H. Neurophysin in the hypothalamoneurohypophysial system. II. Immunocytochemical studies of the ontogeny of oxytocinergic and vasopressinergic neurons. J Neurosci. 1985;5(1):98-109.

41. Dunn KW, Kamocka MM, McDonald JH. A practical guide to evaluating colocalization in biological microscopy. Am J Physiol, Cell Physiol. 2011;300(4):C723-C742.

42. Ito M, Mori Y, Oiso Y, Saito H. A single base substitution in the coding region for neurophysin II associated with familial central diabetes insipidus. JClin Invest. 1991;87(2):725-728.

43. Si-Hoe SL, et al. Endoplasmic reticulum derangement in hypothalamic neurons of rats expressing a familial neurohypophyseal diabetes insipidus mutant vasopressin transgene. FASEB J. 2000;14(12):1680-1684.

44. Colombo C, et al. Seven mutations in the human insulin gene linked to permanent neonatal/ infancy-onset diabetes mellitus. J Clin Invest. 2008;118(6):2148-2156.

45. Nagasaki $\mathrm{H}$, et al. Two novel mutations in the coding region for neurophysin-II associated with familial central diabetes insipidus. JClin Endocrinol Metab. 1995;80(4):1352-1356.
46. Sun S, et al. Epithelial Sel1L is required for the maintenance of intestinal homeostasis. Mol Biol Cell. 2016;27(3):483-490.

47. Wu T, et al. Hrd1 suppresses Nrf2-mediated cellular protection during liver cirrhosis. Genes Dev. 2014;28(7):708-722.

48. Yang H, Qiu Q, Gao B, Kong S, Lin Z, Fang D. Hrd1-mediated BLIMP-1 ubiquitination promotes dendritic cell MHCII expression for CD4 $\mathrm{T}$ cell priming during inflammation. J Exp Med. 2014;211(12):2467-2479.

49. Russell TA, et al. A murine model of autosomal dominant neurohypophyseal diabetes insipidus reveals progressive loss of vasopressin-producing neurons. JClin Invest. 2003;112(11):1697-1706.

50. Hayashi M, et al. Progressive polyuria without vasopressin neuron loss in a mouse model for familial neurohypophysial diabetes insipidus. Am J Physiol Regul Integr Comp Physiol. 2009;296(5):R1641-R1649.

51. Ogen-Shtern N, Ben David T, Lederkremer GZ. Protein aggregation and ER stress. Brain Res. 2016;1648(Pt B):658-666.

52. Travers KJ, Patil CK, Wodicka L, Lockhart DJ, Weissman JS, Walter P. Functional and genomic analyses reveal an essential coordination between the unfolded protein response and ER-associated degradation. Cell. 2000;101(3):249-258.

53. Yoshida H, Matsui T, Hosokawa N, Kaufman RJ, Nagata K, Mori K. A time-dependent phase shift in the mammalian unfolded protein response. Dev Cell. 2003;4(2):265-271.

54. Kaneko M, et al. A different pathway in the endoplasmic reticulum stress-induced expression of human HRD1 and SEL1 genes. FEBS Lett. 2007;581(28):5355-5360.

55. Kosuri P, et al. Protein folding drives disulfide formation. Cell. 2012;151(4):794-806.

56. Di Jeso B, et al. Mixed-disulfide folding intermediates between thyroglobulin and endoplasmic reticulum resident oxidoreductases ERp57 and protein disulfide isomerase. Mol Cell Biol. 2005;25(22):9793-9805.

57. Stefani M, Dobson CM. Protein aggregation and aggregate toxicity: new insights into protein folding, misfolding diseases and biological evolution. JMol Med. 2003;81(11):678-699.

58. Hayashi M, et al. Progressive polyuria without vasopressin neuron loss in a mouse model for familial neurohypophysial diabetes insipidus. Am J Physiol Regul Integr Comp Physiol. 2009;296(5):R1641-R1649. 